\title{
A RECOVERY OF BROUNCKER'S PROOF FOR THE QUADRATURE CONTINUED FRACTION
}

\author{
Sergey KhrushChev
}

\begin{abstract}
350 years ago in Spring of 1655 Sir William Brouncker on a request by John Wallis obtained a beautiful continued fraction for $4 / \pi$. Brouncker never published his proof. Many sources on the history of Mathematics claim that this proof was lost forever. In this paper we recover the original proof from Wallis' remarks presented in his "Arithmetica Infinitorum". We show that Brouncker's and Wallis' formulas can be extended to MacLaurin's sinusoidal spirals via related Euler's products. We derive Ramanujan's formula from Euler's formula and, by using it, then show that numerators of convergents of Brouncker's continued fractions coincide up to a rotation with Wilson's orthogonal polynomials corresponding to the parameters $a=0, b=1 / 2, c=d=1 / 4$.
\end{abstract}

\section{Wallis' product $[22], 1655$}

The first formula for infinite products

$$
\frac{2}{\pi}=\frac{\sqrt{2}}{2} \cdot \frac{\sqrt{2+\sqrt{2}}}{2} \cdot \frac{\sqrt{2+\sqrt{2+\sqrt{2}}}}{2} \cdot \ldots
$$

was found by Viète [21, 1593]. A disadvantage of Viète's formula is that it represents $\pi$ as an infinite product of algebraic irrationalities. In his classical treaty $[\mathbf{2 2}]$ Wallis obtained another formula

$$
\frac{2}{\pi}=\frac{1 \cdot 3}{2 \cdot 2} \cdot \frac{3 \cdot 5}{4 \cdot 4} \cdot \frac{5 \cdot 7}{6 \cdot 6} \cdot \ldots \cdot \frac{(2 n-1) \cdot(2 n+1)}{2 n \cdot 2 n} \cdot \ldots,
$$

in which all multipliers are rational. It was really a great achievement. For instance, Wallis' formula was quite helpful for the Quadrature Problem. Due to Euler's efforts, the ideas derived from Wallis' formula finally

2000 Mathematics Subject Classification. 30B70 (primary), 01A45, 33B15, 33C47, 33D45.

Key words. Continued fractions, infinite products, orthogonal polynomials, special functions. 
resulted in the Lambert-Legendre proof of the irrationality of $\pi$. Euler's analysis of Wallis' proof led him to formulas for the gamma [2, p. 4] and beta functions, as well as to other important discoveries especially in the theory of Continued Fractions.

The standard modern proof of (2) is in fact Euler's improvement (see [9, Ch. IX, §356]) of Wallis' original arguments. Notice that in 1650-56, when Wallis worked on his book, neither the integration by parts nor the change of variable formula was known. Wallis made all his discoveries using a simple relation of integrals with areas and an original method of interpolation. Since $y=\sqrt{1-x^{2}}$ is the equation of the circular arc in the upper half-plane,

$$
\frac{\pi}{4}=\int_{0}^{1} \sqrt{1-x^{2}} d x
$$

is the formula for the area of the quarter of the unit disc. Motivated by Viète's formula, Wallis introduced a family $I(p, q)$ of the reciprocals to the integrals, which he was able to compute

$$
\frac{1}{\int_{0}^{1}\left(1-x^{1 / p}\right)^{q} d x}=\frac{(p+1)(p+2) \ldots(p+q)}{1 \cdot 2 \cdot \ldots \cdot q}=\left(\begin{array}{c}
p+q \\
p
\end{array}\right) .
$$

Here $p$ and $q$ are positive integers. Then, using his interpolation, Wallis found the representation of $I(1 / 2,1 / 2)=4 / \pi$ as an infinite product and obtained (2) from it. A detailed account on Wallis' logic in obtaining (2) is presented in [14] (see a brief version in [13]). A very recent coverage of (2) can be found in the English translation [22, pp. xvii-xx] of "Arithmetica Infinitorum" by J. A. Stedall.

It is interesting that later Euler (see [8, §158]) discovered another proof of Wallis' formula which is based on the development of $\sin (x) / x$ (which plays a crucial role in the proof of Viète's formula) into the infinite product

$$
\frac{\sin x}{x}=\prod_{k=1}^{\infty}\left(1-\frac{x^{2}}{k^{2} \pi^{2}}\right)
$$

Putting here $x=\pi / 2$, Euler derived [8, $§ 185]$ Wallis' formula

$$
\frac{2}{\pi}=\prod_{k=1}^{\infty}\left(1-\frac{1}{(2 k)^{2}}\right)=\prod_{k=1}^{\infty}\left(1-\frac{1}{2 k}\right)\left(1+\frac{1}{2 k}\right)=\prod_{k=1}^{\infty} \frac{(2 k-1)(2 k+1)}{2 k \cdot 2 k} .
$$


With this method Euler obtained Wallis' type formulas for the reciprocal of the length of the side of a square inscribed into the unit circle $\mathbb{T}$

$$
\frac{1}{\sqrt{2}}=\frac{1 \cdot 3}{2^{2}} \cdot \frac{5 \cdot 7}{6^{2}} \cdot \frac{9 \cdot 11}{10^{2}} \cdot \frac{13 \cdot 15}{14^{2}} \cdot \ldots
$$

and

$n \sin \left(\frac{\pi}{2 n}\right)=\frac{(2 n-1) \cdot(2 n+1)}{n \cdot 3 n} \cdot \frac{(4 n-1) \cdot(4 n+1)}{3 n \cdot 5 n} \cdot \frac{(6 n-1) \cdot(6 n+1)}{5 n \cdot 7 n} \cdot \ldots$

for the quarter of the perimeter of a regular $2 n$-polygon inscribed in $\mathbb{T}$.

\section{Brouncker's continued fraction [22], 1655}

According to J. Stedall [22, pp. xviii-xix] the formula (2) appeared between February 28 and July 19, 1655 (when Wallis finished his book) after Wallis' inquiry to Brouncker. In April, 1655 [22, p. xx] Wallis responded to Hobb's threats to reveal his quadrature of the unit circle by publishing the preliminary results of his book. Therefore it is natural to suppose that Wallis' as well as Brouncker's formula were proved in March, 1655. Stedall's opinion that Wallis could prove his formula only after Brouncker showed him his continued fraction

$$
\frac{4}{\pi}=1+\frac{1^{2}}{2}+\frac{3^{2}}{2}+\frac{5^{2}}{2}+\cdots=1+\underset{n=1}{\mathbf{K}}\left(\frac{(2 n-1)^{2}}{2}\right)
$$

seems to be correct. This opinion is also supported by comments given by Wallis to Brouncker's proof of (5), by Wallis' proof of (2), which contains rudiments of the interlacing property of even and odd convergents for continued fractions and which looks quite different from the rest of the book, and also by the fact that Wallis' formula can be derived from Brouncker's (see the end of Section 3). However, there is no doubt that, afterwards, Wallis found his own proof of (2), which later was generalized by Euler (we consider it in Section 4).

Since Brouncker didn't publish his proof, Wallis presented at the end of his book some rather vague comments with his understanding of Brouncker's ideas. This resulted in a positive outcome, however, since the incomplete proof of Brouncker's formula (5) attracted Euler's attention and he wrote two very important papers $[\mathbf{6}]$ and $[\mathbf{7}]$ on analytic properties of Continued Fractions. In Section 17 of [7] Euler clearly indicates that the motivation for this research was to recover Brouncker's proof, which he couldn't complete (see Section 19 of [7]). Instead Euler found his own proofs. Still Euler stressed the importance of the recovery of Brouncker's arguments. As in a great number of other cases Euler's prediction was correct. It is clear from Section 3 of the present paper that 
Brouncker's proof is closely related to the Stieltjes theory of continued fractions, which appeared only in $[\mathbf{1 9}, 1894]$. One can say, as a result, that Analytic Theory of Continued Fractions began from Brouncker's formula.

If one knows that (5) holds, then easy arguments known to Brouncker lead to a proof. Let $P_{n} / Q_{n}, n=-1,0, \ldots, P_{-1}=1, Q_{-1}=0$ be the convergents to (5). Then, the Euler-Wallis formulas (also known as a three-term recurrence, see [10, Ch. $2, \S 2.1 .1]$ ) say that

$$
\begin{aligned}
P_{n} & =2 P_{n-1}+(2 n-1)^{2} P_{n-2} ; \\
Q_{n} & =2 Q_{n-1}+(2 n-1)^{2} Q_{n-2} .
\end{aligned}
$$

These formulas can be rewritten as follows

$$
Q_{n}-(2 n+1) Q_{n-1}=-(2 n-1)\left\{Q_{n-1}-(2 n-1) Q_{n-2}\right\} .
$$

Since $P_{1}=3$ and $P_{2}=2 \cdot 3+9=15$, we obtain that $P_{2}-5 P_{1}=0$ implying by induction that $P_{n}-(2 n+1) P_{n-1}=0$. Observing that $P_{0}=1$, we obtain

$$
P_{n}=1 \cdot 3 \cdot 5 \cdot \ldots \cdot(2 n+1)=(2 n+1) ! ! .
$$

The equalities $Q_{2}=13$ and $Q_{1}=2$ imply that $Q_{2}-5 Q_{1}=3$ and

$$
Q_{n}-(2 n+1) Q_{n-1}=(-1)^{n}(2 n-1) ! !
$$

or equivalently

$$
\frac{Q_{n}}{(2 n+1) ! !}-\frac{Q_{n-1}}{(2 n-1) ! !}=\frac{(-1)^{n}}{(2 n+1)} .
$$

Taking into account (6), we arrive to the formula

$$
\frac{Q_{n}}{P_{n}}=\sum_{k=0}^{n} \frac{(-1)^{k}}{2 k+1}
$$

saying that the convergents of Brouncker's continued fraction coincide with the reciprocals to partial sums of the following alternating series

$$
\frac{\pi}{4}=\sum_{k=0}^{\infty} \frac{(-1)^{k}}{2 k+1}=\int_{0}^{1} \frac{d x}{1+x^{2}}
$$

It is not clear whether Brouncker knew the above formula. However, by at least 1657 , i.e. approximately by the time when Wallis completed his treaty, he definitely knew a similar one (see [13, p. 158])

$$
\ln 2=\sum_{n=1}^{\infty} \frac{1}{(2 n-1) \cdot 2 n}=\sum_{k=1}^{\infty} \frac{(-1)^{k-1}}{k}=\int_{1}^{2} \frac{d x}{x},
$$


which he published with a rigorous proof in [3]. Moreover, according to the report [4] there is evidence that Brouncker knew this formula already in 1654 a year before he learned the problem from Wallis.

The parallel between these two cases becomes more clear if we transform the main trick used in the above computations into the following theorem.

Theorem 1. Let $a>0$ and $\left\{y_{n}\right\}_{n} \geqslant 0$ be a sequence such that $y_{0}>0$, $y_{n}>a$ for $n=1,2, \ldots$ and

$$
\sum_{n=1}^{\infty} \frac{1}{y_{n}}=+\infty
$$

Let $p_{n}=\left(y_{n}-a\right) y_{n-1}, n=1,2, \ldots$. Then

$$
y_{0}+\underset{n=1}{\stackrel{\infty}{\mathbf{K}}}\left(\frac{p_{n}}{a}\right)=\frac{y_{0}}{1+\sum_{n=1}^{\infty}(-1)^{n} \frac{\left(y_{1}-a\right) \ldots\left(y_{n}-a\right)}{y_{1} \ldots y_{n}}} .
$$

Proof: By the Euler-Wallis formulas $P_{n}-y_{n} P_{n-1}=\left(a-y_{n}\right)\left\{P_{n-1}-\right.$ $\left.y_{n-1} P_{n-2}\right\}$. Since $P_{0}-y_{0} P_{1}=0$, this implies $P_{n}=y_{n} P_{n-1}$. Hence $P_{n}=y_{n} y_{n-1} \ldots y_{1} y_{0}$. Similarly

$Q_{n}-y_{n} Q_{n-1}=\left(a-y_{n}\right) \ldots\left(a-y_{1}\right)\left(Q_{0}-y_{0} Q_{1}\right)=\left(a-y_{n}\right) \ldots\left(a-y_{1}\right)$.

It follows that

$$
\frac{Q_{n}}{P_{n}}-\frac{Q_{n-1}}{P_{n-1}}=(-1)^{n} \frac{\left(y_{n}-a\right) \ldots\left(y_{1}-a\right)}{y_{n} y_{n-1} \ldots y_{1} y_{0}},
$$

implying the identity

$$
\frac{Q_{n}}{P_{n}}=\frac{1}{y_{0}}+\frac{1}{y_{0}} \sum_{k=1}^{n}(-1)^{k}\left(1-\frac{a}{y_{k}}\right) \ldots\left(1-\frac{a}{y_{1}}\right) .
$$

The telescopic series in the right-hand side of the above formula converges if and only if

$$
\lim _{n}\left(1-\frac{a}{y_{n}}\right) \ldots\left(1-\frac{a}{y_{1}}\right)=0,
$$

which is the case since $\sum_{n} 1 / y_{n}=+\infty$. 
If $a=2$ and $y_{0}=1, y_{n}=2 n+1$, then $p_{n}=\left(y_{n}-a\right) y_{n-1}=(2 n-1)^{2}$ and we obtain Brouncker's formula:

$$
\begin{aligned}
1+{\underset{K}{K}}_{n}^{\infty}\left(\frac{(2 n-1)^{2}}{2}\right) & =\frac{1}{1+\sum_{n=1}^{\infty}(-1)^{n} \frac{1 \cdot 3 \cdot \ldots \cdot(2 n-1)}{3 \cdot \ldots \cdot(2 n+1)}} \\
& =\frac{1}{\sum_{n=0}^{\infty} \frac{(-1)^{n}}{2 n+1}}=\frac{4}{\pi} .
\end{aligned}
$$

If $a=1, y_{0}=1, y_{n}=n+1$, then $p_{n}=\left(y_{n}-a\right) y_{n-1}=n^{2}$ and

$$
\begin{aligned}
1+\stackrel{\mathbf{K}}{n=1}_{n}\left(\frac{n^{2}}{1}\right) & =\frac{1}{1+\sum_{n=1}^{\infty}(-1)^{n} \frac{1 \cdot 2 \cdot \ldots \cdot n}{2 \cdot \ldots \cdot(n+1)}} \\
& =\frac{1}{\sum_{n=1}^{\infty} \frac{(-1)^{n-1}}{n}}=\frac{1}{\ln 2} .
\end{aligned}
$$

If $a=2, y_{0}=2, y_{n}=n+2$, then $p_{n}=\left(y_{n}-a\right) y_{n-1}=n(n+1)$ for $n=1,2, \ldots$ and

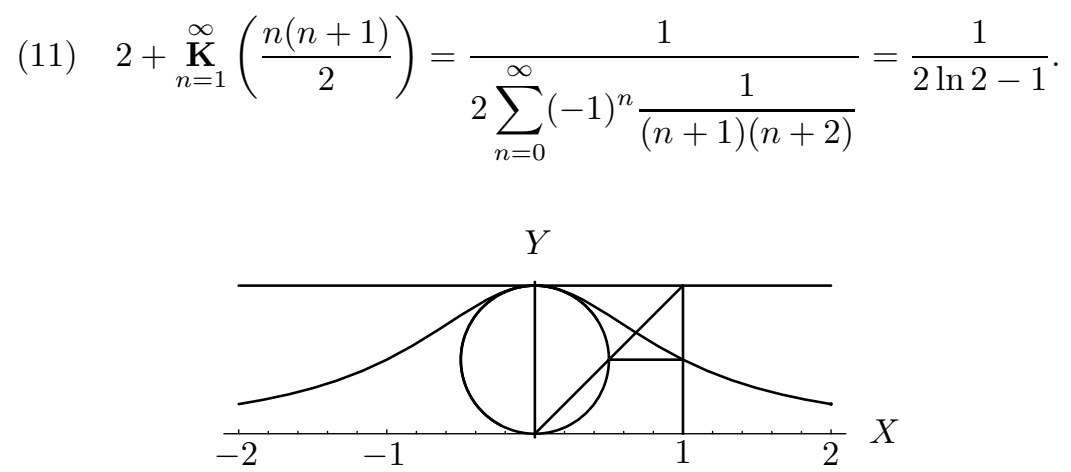

Figure 1. Witch of Agnesi.

Let us return back to (8). The left-hand side of (8) is $\pi / 4$, which is nothing but the quarter of the area of the unit disc or equivalently the area of the disc of diameter 1 (see Figure 1). The right-hand side equals 
the area under the curve $y=1 /\left(1+x^{2}\right)$ bounded by the coordinate axis $O X$ and two vertical lines $x=0$ and $x=1$ (Figure 1 ). The curve $y=1 /\left(1+x^{2}\right)$ has a name. It is called the witch of Agnesi. The Italian mathematician Maria Agnesi included properties of this curve (see Figure 1) in her famous book $[\mathbf{1}, 1748]$ on Analytic Geometry. Known historical materials witness that the first study of this curve was done by Fermat in 1666 ten years after Wallis published his treaty. One can easily find many interesting facts on the witch of Agnesi in the Internet.

To prove Brouncker's formula with the tools, which were available by 1655 , we need the equality of the area of the disc with radius $1 / 2$ to the area under the witch of Agnesi above $0 \leqslant x \leqslant 1$. A direction how to prove this is given by the so-called Pythagorean triples, which were well-known both to Brouncker and Fermat, see [5].

Definition 2. A triple $\{x, y, z\}$ of nonnegative integers is called Pythagorean if it is a solution to the Diophantine equation

$$
x^{2}+y^{2}=z^{2} .
$$

A well-know example of a Pythagorean triple is $\{3,4,5\}$. Pythagorean triples give a complete list of points with rational coordinates on the part of the unit circle in the first quadrant. Other rational points are obtained by symmetries. For instance, $(3 / 5,4 / 5)$ is placed on the unit circle. The rational points on an circular arc can be listed by the rational parametrization of $\mathbb{T}$

$$
x(t)=\frac{2 t}{1+t^{2}}, \quad y(t)=\frac{1-t^{2}}{1+t^{2}} .
$$

The arc of interest corresponds to $0 \leqslant t \leqslant 1$. The formulas

$$
\begin{aligned}
& y(t)-y(s)=\frac{1-t^{2}}{1+t^{2}}-\frac{1-s^{2}}{1+s^{2}}=2 \frac{s^{2}-t^{2}}{\left(1+t^{2}\right)\left(1+s^{2}\right)} ; \\
& x(t)-x(s)=\frac{2 t}{1+t^{2}}-\frac{2 s}{1+s^{2}}=2 \frac{(1-t s)(t-s)}{\left(1+t^{2}\right)\left(1+s^{2}\right)}
\end{aligned}
$$

and an elementary identity $(s+t)^{2}+(1-s t)^{2}=\left(1+s^{2}\right)\left(1+t^{2}\right)$ show that the distance between two points $P=P(s)$ and $Q=Q(t)$ on $\mathbb{T}$ corresponding to $0 \leqslant s<t \leqslant 1$ is given by

$$
\operatorname{dist}(P, Q)=2 \frac{t-s}{\sqrt{\left(1+t^{2}\right)\left(1+s^{2}\right)}} .
$$


It follows that the area of the triangle with vertexes at $P(k / n)$, $Q((k+1) / n)$ and at the origin approximately equals

$$
\operatorname{Area}(\triangle O P Q)=\frac{1}{n} \frac{1+o(1)}{\sqrt{\left(1+\frac{k^{2}}{n^{2}}\right)\left(1+\frac{(k+1)^{2}}{n^{2}}\right)}}=\frac{1+o(1)}{1+\frac{k^{2}}{n^{2}}} \cdot \frac{1}{n},
$$

the area of the corresponding rectangle under the witch of Agnesi. Passing to the limit, we obtain the equality of the required areas. The middle part of (8) can be treated with Brouncker's method [3], see [18, pp. 296-298] or [13, pp. 158-161] for the details and for the comments on Brouncker's proof in $[\mathbf{3}]$.

It could be done this way by Brouncker but it wouldn't. Only 82 years later in [6] Euler found a proof to Brouncker's formula following similar arguments. The original Brouncker's proof can be recovered from the notes made by Wallis in [22, Proposition 191, Comment].

\section{Brouncker's proof}

Two observations play a key role in the recovery of Brouncker's proof. The first is the formula, which is not mentioned in Wallis' comments directly,

$$
\text { (12) } \begin{aligned}
& \frac{1 \cdot 3}{2 \cdot 2} \cdot \frac{3 \cdot 5}{4 \cdot 4} \cdot \frac{5 \cdot 7}{6 \cdot 6} \cdot \ldots \cdot \frac{(2 n-1) \cdot(2 n+1)}{2 n \cdot 2 n} \\
= & \frac{1 \cdot 3}{0}+\frac{2 \cdot 2}{0}+\frac{3 \cdot 5}{0}+\frac{4 \cdot 4}{0}+\cdots+\frac{(2 n-1) \cdot(2 n+1)}{0}+\frac{(2 n) \cdot(2 n)}{1}
\end{aligned}
$$

demonstrating a close relationship of products with continued fractions. Since unfortunately any formal infinite continued fraction with identically zero partial denominators diverges, something should be done to make them positive. The later was known to Brouncker, see [22, p. 169, footnote 79].

The second observation is the following remark by Brouncker [22, p. 168]. One look at the left-hand side of (12) is enough to notice that the numerators are the products of the form

$$
s(s+2)=s^{2}+2 s=(s+1)^{2}-1,
$$

$s$ being odd, whereas the denominators are whole squares of even numbers. This suggest an idea to increase $s$ to $y(s)$ and $s+2$ to $y(s+2)$ so that

$$
y(s) y(s+2)=(s+1)^{2} .
$$


According to Wallis' comments this was the main Brouncker's idea. Then to keep (12) valid the zero partial denominators in the right-hand side of (12) must become positive automatically. That is exactly what we need to complete the proof. The fact that $s+1$ is even is also helpful since it may provide necessary cancellations. Now using (13) repeatedly, we may write

$$
\begin{aligned}
y(1) & =\frac{2^{2}}{y(3)}=\frac{2^{2}}{4^{2}} y(5)=\frac{2^{2}}{4^{2}} \frac{6^{2}}{y(7)}=\frac{2^{2}}{4^{2}} \frac{6^{2}}{8^{2}} y(9)=\cdots \\
& =\frac{2^{2}}{4^{2}} \frac{6^{2}}{8^{2}} \frac{10^{2}}{12^{2}} \ldots \frac{(4 n-2)^{2}}{(4 n)^{2}} y(4 n+1) \\
& =\frac{1^{2}}{2^{2}} \frac{3^{2}}{4^{2}} \frac{5^{2}}{6^{2}} \ldots \frac{(2 n-1)^{2}}{(2 n)^{2}} y(4 n+1) \\
& =\frac{1 \cdot 3}{2^{2}} \cdot \frac{3 \cdot 5}{4^{2}} \cdot \frac{5 \cdot 7}{6^{2}} \cdot \ldots \cdot \frac{(2 n-1)(2 n+1)}{(2 n)^{2}} \cdot \frac{y(4 n+1)}{(2 n+1)} .
\end{aligned}
$$

Combined with Wallis' formula this implies

$$
y(1)=\left(\frac{2}{\pi}+o(1)\right) \cdot \frac{y(4 n+1)}{(2 n+1)} .
$$

Since $s+2<y(s+2)$ and $y(s) y(s+2)=(s+1)^{2}$, the following must be true

$$
s<y(s)<\frac{s^{2}+2 s+1}{s+2}=s+\frac{1}{2+s},
$$

which together with (15) imply

$$
y(1)=\lim _{n}\left(\frac{2}{\pi}+o(1)\right) \cdot \frac{y(4 n+1)}{(2 n+1)}=\frac{4}{\pi} .
$$

It remains only to find a formula for $y(s)$.

We arrived to the crucial point of Brouncker's arguments, which by the way shows that contrary to other mathematicians, see [22, p. xxvii], Brouncker understood very clearly the importance of Wallis' interpolation method. Wallis' main observation was that values of functions $f(s)$ represented by analytic formulas can be uniquely recovered (interpolate) from their values $f(n)$ at integer points $n$. Nowadays, when there are uniqueness theorems for functions holomorphic in domains containing the infinity on their boundary, it is just a matter of a more or less routine application of the uniqueness principle. But in 1652-55 it was a revolutionary discovery. Since Euler thoroughly studied "Arithmetica 
Infinitorum", a possibility that he first obtained the great formula (4) by Wallis' interpolation cannot be excluded.

Here Wallis' interpolation is used in quite a different way. Motivated by (16) one can develop $y(s)$ into a series in positive powers of $1 / s$

$$
y(s)=s+c_{0}+\frac{c_{1}}{s}+\frac{c_{2}}{s^{2}}+\frac{c_{3}}{s^{3}}+\cdots,
$$

and find coefficients $c_{2}, c_{3}, \ldots$, inductively from (13). By (16) $c_{0}=0$. To find $c_{1}$ we assume that

$$
y(s)=s+\frac{c_{1}}{s}+O\left(\frac{1}{s^{2}}\right), \quad s \longrightarrow+\infty .
$$

Using (19), one can easily find $c_{1}$ from the equation

$$
s^{2}+2 s+1=y(s) y(s+2)=s^{2}+2 s+2 c_{1}+O\left(\frac{1}{s}\right),
$$

implying that $c_{1}=1 / 2$. It follows that

$$
y(s)=s+\frac{1}{2 s}+O\left(\frac{1}{s^{2}}\right) \quad s \longrightarrow+\infty .
$$

Similarly, elementary calculations show that $c_{2}=0, c_{3}=-9 / 8, c_{4}=0$, $c_{5}=153 / 16, c_{6}=0$ and therefore

$$
y(s)=s+\frac{8 s^{4}-18 s^{2}+153}{16 s^{5}}+O\left(\frac{1}{s^{7}}\right) .
$$

Applying Euclidean Algorithm to the quotient of polynomials, we have

$$
\begin{aligned}
\frac{8 s^{4}-18 s^{2}+153}{16 s^{5}} & =\frac{1}{2 s+\frac{9\left(4 s^{3}-34 s\right)}{8 s^{4}-18 s^{2}+153}}=\frac{1}{2 s+\frac{9}{\frac{8 s^{4}-18 s^{2}+153}{4 s^{3}-34 s}}} \\
& =\frac{1}{2 s+\frac{9}{2 s+\frac{25\left(2 s^{2}+153 / 25\right)}{4 s^{3}-34 s}}}=\frac{1}{2 s+\frac{9}{2 s+\frac{25}{2 s+\cdots}}} .
\end{aligned}
$$

A remarkable property of the above calculations is that $1^{2}=1,3^{2}=9$, $5^{2}=25$, etc appear automatically as common divisors of the coefficients of the polynomials in Euclid's algorithm. The fraction 153/25 appears only because we didn't find $c_{7}$. 
Increasing the number of terms in (18) we naturally arrive to the conclusion that

$$
y(s)=s+\frac{1^{2}}{2 s}+\frac{3^{2}}{2 s}+\frac{5^{2}}{2 s}+\frac{7^{2}}{2 s}+\cdots+\frac{(2 n-1)^{2}}{2 s}+\cdots
$$

Having got (21), we may reverse the order of arguments and compute the differences

$$
\begin{aligned}
\frac{P_{0}(s)}{Q_{0}(s)} \frac{P_{0}(s+2)}{Q_{0}(s+2)}-(s+1)^{2} & =s(s+2)-(s+1)^{2}=(-1)=O(1) ; \\
\frac{P_{1}(s)}{Q_{1}(s)} \frac{P_{1}(s+2)}{Q_{1}(s+2)}-(s+1)^{2} & =\frac{4 s^{4}+16 s^{3}+20 s^{2}+8 s+9}{4 s^{2}+8 s} \\
-\frac{4 s^{4}+16 s^{3}+20 s^{2}+8 s}{4 s^{2}+8 s} & =\frac{9}{4 s^{2}+8 s}=O\left(\frac{1}{s^{2}}\right) ; \\
\frac{P_{2}(s)}{Q_{2}(s)} \frac{P_{2}(s+2)}{Q_{2}(s+2)}-(s+1)^{2} & =\frac{16 s^{6}+96 s^{5}+280 s^{4}+480 s^{3}+649 s^{2}+594 s}{16 s^{4}+64 s^{3}+136 s^{2}+144 s+225} \\
-(s+1)^{2} & =\frac{-225}{16 s^{4}+64 s^{3}+136 s^{2}+144 s+225}=O\left(\frac{1}{s^{4}}\right) .
\end{aligned}
$$

One can find these very formulas in Wallis [1656, pp. 169-170], where Wallis writes after the last formula: "... which is less than the square $F^{2}+2 F+1^{1}$. And thus it may be done as far as one likes; it will form a product which will be (in turn) now grater than, now less than, the given square (the difference, however, continually decreasing, as is clear), which was to be proved".

To make Wallis' comments more clear, we observe that the denominators of the boxed fractions being the products $Q_{n}(s) Q_{n}(s+2)$ of polynomials with positive coefficients, as the Euler-Wallis formulas show, are polynomials with positive coefficients as well. Next, if the polynomial

$$
P_{n}(s) P_{n}(s+2)-(s+1)^{2} Q_{n}(s) Q_{n}(s+2)=b_{n}
$$

is a constant, then $b_{n}=-(-1)^{n}[(2 n+1) ! !]^{2}$. Indeed, for $n=0,1,2$ this can be seen from the above formulas presented by Wallis in his book. If (22) holds, then evaluating it at $s=-1$ we obtain $P_{n}(-1) P_{n}(1)=b_{n}$. Observing that polynomials $P_{n}(s)$ are odd for even $n$ and even for odd $n$

\footnotetext{
${ }^{1}$ In Wallis' notations $s=F$.
} 
and applying (6), we obtain that $b_{n}=-(-1)^{n}[(2 n+1) ! !]^{2}$. Hence for every positive $s$

$$
\frac{P_{2 k}(s)}{Q_{2 k}(s)} \cdot \frac{P_{2 k}(s+2)}{Q_{2 k}(s+2)}<(s+1)^{2}<\frac{P_{2 k+1}(s)}{Q_{2 k+1}(s)} \cdot \frac{P_{2 k+1}(s+2)}{Q_{2 k+1}(s+2)} .
$$

The continued fraction (21) with positive terms converges (see Theorem 15 below). Its even convergents increase to $y(s)$ whereas odd convergents decrease to $y(s)$. Passing to the limit in the above inequalities, we obtain that the continued fraction $y(s)$ satisfies the functional equation (13). The inequality $s<y(s)$ is clear from (21). Thus the proof of Brouncker's formula is completed provided (22) is proved (see Theorem 12 below).

It is interesting that the above arguments invented by Brouncker were independently used much later by Stieltjes in his approach to the Moment Problem [19]. Brouncker knew that regular continued fractions are obtained from the development of real numbers into decimal fractions. Therefore, dealing with quotients of polynomials, as Brouncker did applying Wallis' interpolation, one may try to exploit the analogy between quotients of polynomials and of rational numbers, where $1 / s$ corresponds to $1 / 10$. Then the recovery of $y(s)$ by its asymptotic expansion at the infinity looks completely similar to the recovery of a real number from its decimal representation. In his theory Stieltjes used the same analogy.

The analogy between asymptotic expansions of functions and decimal representations of numbers is fruitful but must be executed with care. Every decimal expansion determines one number, whereas, for instance, zero asymptotic expansion in $1 / s$ at infinity corresponds to a zero function as well as to $e^{-s}$.

This is more or less what Wallis tried to explain in his comments. In view of absence in 1655 of any clear formalism for asymptotic expansions, Wallis' explanations were so obscure. It may also happen that Wallis didn't understand Brouncker's revolutionary ideas to the full extent, since it was not at all easy to recover them from formal writings.

In [18, pp. 300-306] Stedall gives another, purely numerical and algebraic version of Brouncker's possible arguments. One can find in $[\mathbf{1 8}$, p. 302] the references to other attempts to recover the proof of Brouncker's formula.

The first proof of Brouncker formula was given by Euler [7] who used Differential Calculus.

As it has been mentioned, Wallis' formula follows from that of Brouncker. Combining (14) with the arguments of Section 2 and observing that 
$y(1)=4 / \pi$ by (16) one can easily obtain

$$
\begin{gathered}
\frac{1 \cdot 3}{2^{2}} \cdot \frac{3 \cdot 5}{4^{2}} \cdot \frac{5 \cdot 7}{6^{2}} \cdot \ldots \cdot \frac{(2 n-1)(2 n+1)}{(2 n)^{2}} \cdot\left(2-\frac{1}{2 n+1}\right)<y(1) \\
=\frac{4}{\pi}<\frac{1 \cdot 3}{2^{2}} \cdot \frac{3 \cdot 5}{4^{2}} \cdot \frac{5 \cdot 7}{6^{2}} \cdot \ldots \cdot \frac{(2 n-1)(2 n+1)}{(2 n)^{2}} \cdot \frac{4 n+2}{4 n+3} \cdot 2,
\end{gathered}
$$

which obviously implies (2).

\section{Euler's extension of Wallis' formula and sinusoidal spirals}

Motivated by [22] Euler extended Wallis' formula (3) to transcendental quotients of the integrals similar to $I(p, q)$. It was especially attractive to have such an extension, since Brouncker's formula indicated that there might be such generalizations. To get a correct guidance which quotients must be considered, Euler first observed that the product of two integrals in Wallis' proof

$$
\begin{aligned}
& \int_{0}^{1} \frac{x^{2 n}}{\sqrt{1-x^{2}}} d x=\frac{\pi}{2} \cdot \frac{1 \cdot 3 \cdot 5 \cdot \ldots \cdot(2 n-1)}{2 \cdot 4 \cdot 6 \cdot \ldots \cdot 2 n}, \\
& \int_{0}^{1} \frac{x^{2 n+1}}{\sqrt{1-x^{2}}} d x=\frac{2 \cdot 4 \cdot 6 \cdot \ldots \cdot 2 n}{3 \cdot 5 \cdot 7 \cdot \ldots \cdot(2 n+1)}
\end{aligned}
$$

satisfies a simple equation

$$
I(\alpha) \stackrel{\text { def }}{=} \int_{0}^{1} \frac{x^{2 \alpha}}{\sqrt{1-x^{2}}} d x \int_{0}^{1} \frac{x^{2 \alpha+1}}{\sqrt{1-x^{2}}} d x=\frac{1}{2 \alpha+1} \cdot \frac{\pi}{2}
$$

for nonnegative integer $\alpha$. The fact that this formula holds for any $\alpha>-1$ was mentioned by Euler already in [7] and later stated without proof as a lemma in [9, Ch. VIII, §332]. It is more or less clear that Euler considered this obvious since from integration by parts he knew that the function $\alpha \rightarrow(2 \alpha+1) I(\alpha)$ is periodic. On the other hand simple inequalities from Euler's proof of Walis' formula [9, Ch. VIII, $\S 332]$, show that this function has the limit $2 / \pi$ at infinity. Hence it must be equal to $2 / \pi$ everywhere. In these terms Wallis' result looks as follows

$$
\begin{aligned}
& \int_{0}^{1} \frac{x^{2 n} d x}{\sqrt{1-x^{2}}} / \int_{0}^{1} \frac{x^{2 n+1} d x}{\sqrt{1-x^{2}}}=\left[\frac{1 \cdot 3 \cdot 5 \cdot \ldots \cdot(2 n-1)}{2 \cdot 4 \cdot 6 \cdot \ldots \cdot 2 n}\right]^{2} \cdot \frac{\pi}{2}(2 n+1) \\
& \int_{0}^{1} \frac{x^{2 n} d x}{\sqrt{1-x^{2}}} \cdot \int_{0}^{1} \frac{x^{2 n+1} d x}{\sqrt{1-x^{2}}}=\frac{1}{2 n+1} \cdot \frac{\pi}{2}
\end{aligned}
$$


The change of variables $x:=r^{n / 2}$ in (24) followed by the substitution of $\alpha$ with $1 / n-1 / 2>-1 / 2$ transforms (24) into

$$
\int_{0}^{1} \frac{d r}{\sqrt{1-r^{n}}} \times \int_{0}^{1} \frac{r^{n / 2} d r}{\sqrt{1-r^{n}}}=\frac{\pi}{n} .
$$

For every positive $n(26)$ determines a polar curve $r=r(\theta)$ such that its element of length $d s$ satisfies

$$
d s=\sqrt{1+\left(r \frac{d \theta}{d r}\right)^{2}}=\frac{d r}{\sqrt{1-r^{n}}} .
$$

This differential equation in $\theta$ as a function of radius $r$ can easily be integrated to obtain the equation of the corresponding polar curve

$$
r^{n / 2}=\cos \left( \pm \frac{n \theta}{2}+A\right)
$$

where $A$ is a constant. A constant $A$ is responsible for rotations whereas the sign change makes symmetries with respect to real axis. With $A=0$, $n$ rational, and $\pm=+$ the curve $(27)$ is known as sinusoidal spiral. It was first studied by Colin Maclaurin in 1718 for rational values of $n$.

If $n=2, A=\pi / 2$, and the sign is - , then this curve is the circle $r=\sin \theta$ pictured on Figure 1. If $n=4, A=0$, and the sign is + , then we obtain the equation $r^{2}=\cos 2 \theta$ of the lemniscate of Bernoulli presented on Figure 2. It was studied in 1694 by Jakob Bernoulli and can also be defined by the algebraic equation $\left(x^{2}+y^{2}\right)^{2}=x^{2}-y^{2}$.

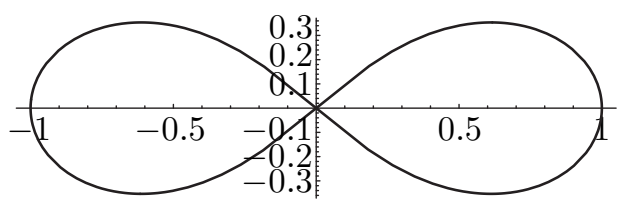

Figure 2. Lemniscate of Bernoulli.

If $n=6, A=0$, and the sign is + , then $r^{3}=\cos 3 \theta$ and we obtain the 3-lemniscate, see Figure 3. For $n=6, A=\pi / 2, \pm=-$, it is called Kiepert's curve or the three-pole lemniscate. It looks very close to the trifolium $r=\cos 3 \theta$.

Since all these curves belong to one family of sinusoidal spirals (27), one may expect that they may correspond to infinite products similar 
to (2) obtained by Wallis for the 1-lemniscate (the unite circle). The choice of $n$ must be rational, since $n$ will definitely enter these formulas.

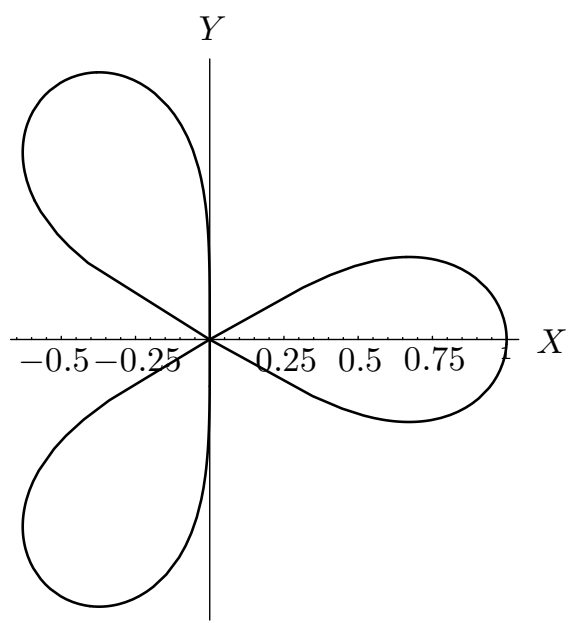

Figure 3. The 3-lemniscate (three-pole lemniscate).

For the circle $(n=2)$ Wallis' formula can be written as

$$
\frac{2}{\pi}=\frac{\int_{0}^{1} \frac{x d x}{\sqrt{1-x^{2}}}}{\int_{0}^{1} \frac{d x}{\sqrt{1-x^{2}}}}=\frac{1 \cdot 3}{2 \cdot 2} \cdot \frac{3 \cdot 5}{4 \cdot 4} \cdot \frac{5 \cdot 7}{6 \cdot 6} \cdot \frac{7 \cdot 9}{8 \cdot 8} \cdot \ldots
$$

Our purpose is to interpolate Wallis' formula to the class of MacLaurin's sinusoidal spirals and obtain infinite rational products for

$$
\frac{\int_{0}^{1} \frac{r^{n / 2} d x}{\sqrt{1-r^{n}}}}{\int_{0}^{1} \frac{d r}{\sqrt{1-r^{n}}}}
$$

For a positive $n, A=0$ and $\pm=+$ in $(27)$ the curve starts at $(1,0)$ and moves to the origin with increase of $\theta$. The denominator in the above formula is the lengtht of this part of the curve. So the whole expression is the mean value of the powered distance $r^{n / 2}$ to the origin taken along the curve against the length. 
The following lemma generalizes another Wallis' formula

$$
\int_{0}^{1}\left(1-x^{2}\right)^{\frac{n}{2}} d x=\frac{n}{n+1} \int_{0}^{1}\left(1-x^{2}\right)^{\frac{n-2}{2}} d x .
$$

Lemma 3 (Euler, [9, Ch. IX, §360]). For positive $m, n, k$

$$
\int_{0}^{1} x^{m-1}\left(1-x^{n}\right)^{\frac{k}{n}-1} d x=\frac{m+k}{m} \int_{0}^{1} x^{m+n-1}\left(1-x^{n}\right)^{\frac{k}{n}-1} d x .
$$

Proof: The formula of the lemma follows from the identity

$$
\begin{aligned}
& \int_{0}^{1} x^{m+n-1}\left(1-x^{n}\right)^{\frac{k}{n}-1} d x=-\frac{1}{k} \int_{0}^{1} x^{m} d\left(1-x^{n}\right)^{\frac{k}{n}} \\
& \quad=\frac{m}{k} \int_{0}^{1} x^{m-1}\left(1-x^{n}\right)^{\frac{k}{n}-1} d x-\frac{m}{k} \int_{0}^{1} x^{m+n-1}\left(1-x^{n}\right)^{\frac{k}{n}-1} d x .
\end{aligned}
$$

Theorem 4 (Euler, [7]). For positive $m, \mu, n$ and $k$

$$
\frac{\int_{0}^{1} x^{m-1}\left(1-x^{n}\right)^{\frac{k-n}{n}} d x}{\int_{0}^{1} x^{\mu-1}\left(1-x^{n}\right)^{\frac{k-n}{n}} d x}=\prod_{j=0}^{\infty} \frac{(\mu+j n)(m+k+j n)}{(\mu+k+j n)(m+j n)}
$$

Proof: The proof of this theorem follows the proof of Wallis' formula. Iterating Lemma 3, we obtain in $s$ steps that

$$
\begin{gathered}
\int_{0}^{1} \frac{x^{m-1} d x}{\left(1-x^{n}\right)^{1-k / n}} d x=u_{s} \cdot \prod_{j=0}^{s-1} \frac{m+k+j n}{m+j n} ; \\
\int_{0}^{1} \frac{x^{\mu-1} d x}{\left(1-x^{n}\right)^{1-k / n}}=v_{s} \cdot \prod_{j=0}^{s-1} \frac{\mu+k+j n}{\mu+j n},
\end{gathered}
$$

where

$$
u_{s}=\int_{0}^{1} \frac{x^{m+s n-1} d x}{\left(1-x^{n}\right)^{1-k / n}} \quad \text { and } \quad v_{s}=\int_{0}^{1} \frac{x^{\mu+s n-1} d x}{\left(1-x^{n}\right)^{1-k / n}} d x .
$$

If $\mu=m$, then there is nothing to prove. Since $\mu$ and $m$ enter the formulas symmetrically, we may assume that $\mu<m$. Since $t \rightarrow x^{t}$ monotonically decreases in $t$ for $0<x<1$, the integration over $(0,1)$ shows that

$$
v_{s}>u_{s}>v_{s+[(m-\mu) / n]+1} .
$$


It follows that

$$
\frac{v_{s}}{u_{s}}>1>\frac{v_{s}}{u_{s}} \frac{v_{s+[(m-\mu) / n]+1}}{v_{s}}=\frac{v_{s}}{u_{s}} w_{s}
$$

where

$$
w_{s}=\prod_{j=s}^{s+[(m-\mu) / n]+1} \frac{\mu+j n}{\mu+k+j n} \longrightarrow 1,
$$

as $s \rightarrow+\infty$. Hence

$$
0<\frac{v_{s}}{u_{s}}-1<\frac{1}{w_{s}}-1=\frac{1-w_{s}}{w_{s}} \longrightarrow 0 .
$$

Notice that Euler's Theorem gives a formula for any convergent product of the form

$$
\prod_{j=0}^{\infty} \frac{(a+j n)(b+j n)}{(c+j n)(d+j n)}
$$

with positive $a, b, c, d$. Indeed, the identity

$$
\frac{(a+j n)(b+j n)}{(c+j n)(d+j n)}=1+\frac{j n(a+b-c-d)+a b-c d}{(c+j n)(d+j n)}
$$

shows that the product converges if and only if $a+b=c+d$, which is equivalent to $c-a=b-d=k$. If $k=0$ then each term of the product is 1 . Is $k<0$, then one can consider the reciprocal of this product and reduce it to the case $k>0$. Hence $c=a+k, b=d+k$. To find the value of the product it remains to put $\mu=a, m=d$ in (29).

Remark. Nowadays these formulas are obtained with a help of the gamma function, see $[\mathbf{2 3}, \S 12 \cdot 13]$. In fact they played a crucial role in the discovery of the gamma function by Euler. The change of variables $t=x^{n}$ in the left hand side of (29) shows that it is the quotient of two beta functions

$$
\frac{\int_{0}^{1} x^{m-1}\left(1-x^{n}\right)^{\frac{k-n}{n}} d x}{\int_{0}^{1} x^{\mu-1}\left(1-x^{n}\right)^{\frac{k-n}{n}} d x}=\frac{B\left(\frac{m}{n}, \frac{k}{n}\right)}{B\left(\frac{\mu}{n}, \frac{k}{n}\right)}
$$

where

$$
B(p, q)=\int_{0}^{1} x^{p-1}(1-x)^{q-1} d x .
$$

The correspondence established in (29) between the pairs of integrals and the products is not one-to-one. For instance, putting in (29) first 
$\mu=p, m=p+r, k=2 q, n=2 r$, and then $\mu=p, m=p+2 q, k=r$, $n=2 r$, we obtain

$$
\begin{aligned}
& \frac{\int_{0}^{1} x^{p+r-1}\left(1-x^{2 r}\right)^{\frac{q}{r}-1} d x}{\int_{0}^{1} x^{p-1}\left(1-x^{2 r}\right)^{\frac{q}{r}-1} d x}=\prod_{j=0}^{\infty} \frac{(p+2 j r)(p+2 q+r+2 j r)}{(p+2 q+2 j r)(p+r+2 j r)} \\
& \frac{\int_{0}^{1} x^{p+2 q-1}\left(1-x^{2 r}\right)^{-\frac{1}{2}} d x}{\int_{0}^{1} x^{p-1}\left(1-x^{2 r}\right)^{-\frac{1}{2}} d x}=\prod_{j=0}^{\infty} \frac{(p+2 j r)(p+2 q+r+2 j r)}{(p+2 q+2 j r)(p+r+2 j r)} .
\end{aligned}
$$

Formula (31) with $p=1, q=1 / 2, r=1$, is Wallis' formula (28). If $p=1$, $q=1, r=2$ in (31), then

$$
\frac{\int_{0}^{1} \frac{x^{2} d x}{\sqrt{1-x^{4}}}}{\int_{0}^{1} \frac{d x}{\sqrt{1-x^{4}}}}=\frac{1 \cdot 5}{3 \cdot 3} \cdot \frac{5 \cdot 9}{7 \cdot 7} \cdot \frac{9 \cdot 13}{11 \cdot 11} \cdot \frac{13 \cdot 17}{15 \cdot 15} \cdot \ldots
$$

is Euler's infinite product for the moment of inertia of the unit mass uniformly distributed along the lemniscate of Bernoulli. With $n=4$ formula (26) turns into another Euler's formula, see [7] or [9, p. 185]

$$
\int_{0}^{1} \frac{d x}{\sqrt{1-x^{4}}} \times \int_{0}^{1} \frac{x^{2} d x}{\sqrt{1-x^{4}}}=\frac{\pi}{4}
$$

called the lemniscate identity $[\mathbf{1 5}$, p. 69]. For the 3-lemniscate we have

$$
\frac{\int_{0}^{1} \frac{x^{3} d x}{\sqrt{1-x^{6}}}}{\int_{0}^{1} \frac{d x}{\sqrt{1-x^{6}}}}=\frac{1 \cdot 7}{4^{2}} \cdot \frac{7 \cdot 13}{10^{2}} \cdot \frac{13 \cdot 19}{16^{2}} \cdot \frac{19 \cdot 25}{22^{2}} \cdot \ldots
$$

For the $n$-lemniscate $r^{n / 2}=\cos (n \theta / 2)$ with $p=1, q=n / 4, r=n / 2$, in $(31)$

$$
\frac{\int_{0}^{1} \frac{x^{n / 2} d x}{\sqrt{1-x^{n}}}}{\int_{0}^{1} \frac{d x}{\sqrt{1-x^{n}}}}=\prod_{j=0}^{\infty} \frac{(1+j n)(1+(j+1) n)}{(1+n / 2+j n)^{2}} .
$$




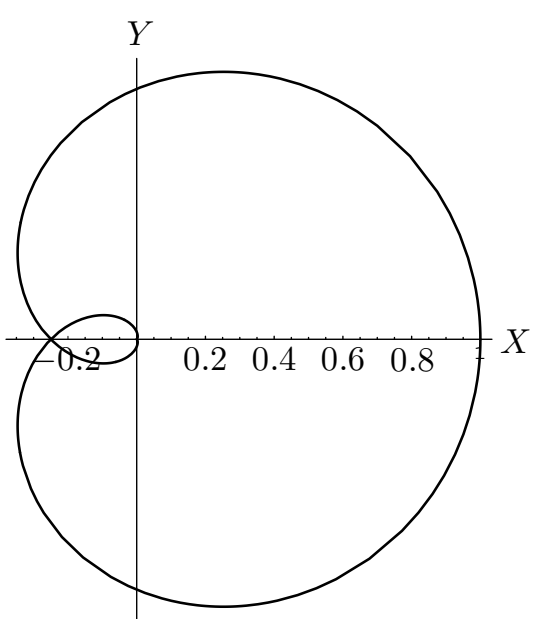

Figure 4. The 1/4-lemniscate.

In particular for $n=1$ (cardioid)

$(35)$

$$
\begin{aligned}
\frac{\int_{0}^{1} \frac{x^{1 / 2} d x}{\sqrt{1-x}}}{\int_{0}^{1} \frac{d x}{\sqrt{1-x}}} & =\frac{2 \cdot 4}{3^{2}} \cdot \frac{4 \cdot 6}{5^{2}} \cdot \frac{6 \cdot 8}{7^{2}} \cdot \frac{8 \cdot 10}{9^{2}} \cdot \ldots \\
& =\frac{1}{2} \cdot \frac{2^{2}}{1 \cdot 3} \cdot \frac{4^{2}}{3 \cdot 5} \cdot \frac{6^{2}}{5 \cdot 7} \cdot \ldots=\frac{\pi}{4}
\end{aligned}
$$

see (2). For $n=1 / 2($ the $1 / 4$-lemniscate, see Figure 4$)$,

$$
\frac{9 \pi}{32}=\frac{\int_{0}^{1} \frac{x^{1 / 4} d x}{\sqrt{1-\sqrt{x}}}}{\int_{0}^{1} \frac{d x}{\sqrt{1-\sqrt{x}}}}=\frac{4 \cdot 6}{5^{2}} \cdot \frac{6 \cdot 8}{7^{2}} \cdot \frac{8 \cdot 10}{9^{2}} \cdot \frac{10 \cdot 12}{11^{2}} \cdot \ldots
$$

which again resembles (2). This has a simple explanation. If $m$ is a positive integer and $n=1 / m$, then the change of variables $x:=x^{2 m}$ and 
Lemma 3 show that

$$
\begin{aligned}
& \int_{0}^{1} \frac{x^{n / 2} d x}{\sqrt{1-x^{n}}}=2 m \int_{0}^{1} \frac{x^{2 m} d x}{\sqrt{1-x^{2}}} \\
& \int_{0}^{1} \frac{d x}{\sqrt{1-x^{n}}}=2 m \int_{0}^{1} \frac{x^{2 m-1} d x}{\sqrt{1-x^{2}}}=\frac{2 m+1}{2 m} \int_{0}^{1} \frac{x^{2 m+1} d x}{\sqrt{1-x^{2}}} .
\end{aligned}
$$

Then by (25)

$$
\text { (36) } \begin{aligned}
& \frac{\int_{0}^{1} \frac{x^{n / 2} d x}{\sqrt{1-x^{n}}}}{\int_{0}^{1} \frac{d x}{\sqrt{1-x^{n}}}}=\frac{1 \cdot 3}{2^{2}} \cdot \frac{3 \cdot 5}{4^{2}} \cdot \ldots \cdot \frac{(2 m-1) \cdot(2 m+1)}{(2 m)^{2}} \cdot \frac{2 m}{2 m+1} \cdot \frac{\pi}{2} \\
& =\frac{(2 m)(2 m+2)}{(2 m+1)^{2}} \cdot \frac{(2 m+2)(2 m+4)}{(2 m+3)^{2}} \cdot \frac{(2 m+4)(2 m+6)}{(2 m+5)^{2}} \cdot \ldots .
\end{aligned}
$$

Therefore essentially new formulas may be related with sinusoidal spirals corresponding to integer values of $n, n>2$.

Identity (26) may be used to obtain infinite products for the lengths of sinusoidal spirals. If $n=2 r$, then by (26) and (31)

$$
\begin{aligned}
\int_{0}^{1} \frac{d x}{\sqrt{1-x^{2 r}}}=\frac{\pi / 2 r}{\int_{0}^{1} \frac{x^{r} d x}{\sqrt{1-x^{2 r}}}}= & \frac{\int_{0}^{1} \frac{x^{r-1} d x}{\sqrt{1-x^{2 r}}}}{\int_{0}^{1} \frac{x^{r} d x}{\sqrt{1-x^{2 r}}}} \\
& =\prod_{j=0}^{\infty} \frac{(r+1+2 j r)(2 r+2 r j)}{(r+2 j r)(2 r+1+2 j r)}
\end{aligned}
$$

For example, the lemniscate of Bernoulli $(r=2)$ is made of four equal arcs (Figure 2) with length

$$
L_{1}=\int_{0}^{1} \frac{d x}{\sqrt{1-x^{4}}}=\prod_{j=0}^{\infty} \frac{(3+4 j)(4+4 j)}{(2+4 j)(5+4 j)}=1.311028777 \ldots
$$

each. To obtain an analogue of Wallis' formula for the $r$-lemniscate we rewrite (37) as follows

$$
\frac{1}{\int_{0}^{1} \frac{d x}{\sqrt{1-x^{2 r}}}}=\frac{1 \cdot(2 r+1)}{2 \cdot(r+1)} \cdot \frac{3 \cdot(4 r+1)}{4 \cdot(3 r+1)} \cdot \frac{5 \cdot(6 r+1)}{6 \cdot(5 r+1)} \cdot \ldots,
$$


which obviously gives Wallis' formula if $r=1$. For the cardioid $(r=1 / 2)$ it turns into

$$
1=\frac{1 \cdot 2}{1 \cdot 3} \cdot \frac{3 \cdot 3}{2 \cdot 5} \cdot \frac{5 \cdot 4}{3 \cdot 7} \cdot \frac{7 \cdot 5}{4 \cdot 9} \cdot \frac{9 \cdot 6}{5 \cdot 11} \cdot \ldots
$$

and for the 1/4-lemniscate, Figure 4, it gives a more interesting formula

$$
\frac{3}{8}=\frac{1 \cdot 3}{1 \cdot 5} \cdot \frac{3 \cdot 4}{2 \cdot 7} \cdot \frac{5 \cdot 5}{3 \cdot 9} \cdot \frac{7 \cdot 6}{4 \cdot 11} \cdot \frac{9 \cdot 7}{5 \cdot 13} \cdot \frac{11 \cdot 8}{6 \cdot 15} \cdot \ldots
$$

\section{General Brouncker's continued fraction and Euler's formula}

As soon as the motivation for constructing Brouncker continued fraction is clarified, the proof of (5) can be completed as indicated in Section 2. However, in fact Brouncker proved more than (5). His initial idea to construct $y(s)$ satisfying both $s<y(s)$ and the functional equation (13) resulted in a remarkable and important identity valid for $s>1$

$$
\text { (40) } \begin{aligned}
s^{2}=((s-1) & \left.+\frac{1^{2}}{2(s-1)}+\frac{3^{2}}{2(s-1)}+\frac{5^{2}}{2(s-1)}+\cdots\right) \\
& \times\left((s+1)+\frac{1^{2}}{2(s+1)}+\frac{3^{2}}{2(s+1)}+\frac{5^{2}}{2(s+1)}+\cdots\right) .
\end{aligned}
$$

The Algebraic identity (13), which finally led to (21), can easily be used to represent $y(s)$ as an infinite product.

Theorem 5. Let $y(s)$ be a function on $(0,+\infty)$ satisfying the functional equation (13) and the inequality $s<y(s)$ for $s>C$, where $C$ is some constant. Then

$$
y(s)=(s+1) \prod_{n=1}^{\infty} \frac{(s+4 n-3)(s+4 n+1)}{(s+4 n-1)^{2}},
$$

$y(s)$ extends to a holomorphic function in the whole complex plane except for the points $s=-3,-7,-11, \ldots$, where $y(s)$ has simple poles, and $y(s)$ satisfies (13) for all complex $s$. 
Proof: Iterating (13), we obtain

$$
\begin{aligned}
y(s) & =\frac{(s+1)^{2}}{(s+3)^{2}} y(s+4)=\frac{(s+1)^{2}}{(s+3)^{2}} \cdot \frac{(s+5)^{2}}{(s+7)^{2}} y(s+8) \\
& =\frac{(s+1)^{2}}{(s+3)^{2}} \cdot \frac{(s+5)^{2}}{(s+7)^{2}} \cdot \ldots \cdot \frac{(s+4 n-3)^{2}}{(s+4 n-1)^{2}} y(s+4 n) \\
& =(s+1) \frac{(s+1)(s+5)}{(s+3)^{2}} \cdot \ldots \cdot \frac{(s+4 n-3)(s+4 n+1)}{(s+4 n-1)^{2}} \frac{y(s+4 n)}{(s+4 n+1)} .
\end{aligned}
$$

Multipliers are grouped in accordance to the rule of Wallis' formula:

$$
\frac{(s+4 n-3)(s+4 n+1)}{(s+4 n-1)^{2}}=1-\frac{4}{(s+4 n-1)^{2}},
$$

which implies the convergence of the infinite product for $s \neq-3,-7, \ldots$ to a holomorphic function. To show that the infinite product converges to $y(s)$ for $s>0$, we must check that

$$
\lim _{n} \frac{y(s+4 n)}{(s+4 n+1)}=1 .
$$

Since $y(s)$ satisfies (13), we obtain that

$$
s+4 n<y(s+4 n)=\frac{(s+4 n+1)^{2}}{y(s+4 n-2)}<\frac{(s+4 n+1)^{2}}{(s+4 n-2)},
$$

which obviously implies what we need.

Corollary 6 (Brouncker). For positive s we have

$$
y(s)=(s+1) \prod_{n=1}^{\infty} \frac{(s+4 n-3)(s+4 n+1)}{(s+4 n-1)^{2}}=s+{\underset{K=1}{\mathbf{K}}}_{n=1}\left(\frac{(2 n-1)^{2}}{2 s}\right),
$$

where $y(s)>s$ and satisfies $y(s) y(s+2)=(s+1)^{2}$.

Following the ideology of "Arithmetica Infinitorum" this corollary interpolates Wallis' result $(s=1)$ to the whole scale of positive $s$. In [7] Euler explicitly stated and proved Corollary 6. His proof, however, was rather complicated and also required some restoration. We need a simple exemption from this proof.

Corollary 7 (Euler, [7]). For $s>0$

$$
s+\underset{n=1}{\infty}\left(\frac{(2 n-1)^{2}}{2 s}\right)=(s+1) \frac{\int_{0}^{1} \frac{x^{s+2} d x}{\sqrt{1-x^{4}}}}{\int_{0}^{1} \frac{x^{s} d x}{\sqrt{1-x^{4}}}} .
$$


Proof: Formula (29) with $n=4, k=2, m=s+3, \mu=s+1$ implies that

$$
\begin{aligned}
\frac{\int_{0}^{1} \frac{x^{s+2} d x}{\sqrt{1-x^{4}}}}{\int_{0}^{1} \frac{x^{s} d x}{\sqrt{1-x^{4}}}} & =\prod_{j=0}^{\infty} \frac{(s+1+4 j)(s+5+4 j)}{(s+3+4 j)^{2}} \\
& =\prod_{n=1}^{\infty} \frac{(s+4 n-3)(s+4 n+1)}{(s+4 n-1)^{2}}
\end{aligned}
$$

which proves the corollary.

Wallis' integrals (29) relate the values of $y(s)$ at rational points with sinusoidal spirals:

$$
\begin{aligned}
& \frac{4}{n} \cdot \frac{\int_{0}^{1} \frac{r^{n / 2} d r}{\sqrt{1-r^{n}}}}{\int_{0}^{1} \frac{d r}{\sqrt{1-r^{n}}}} \stackrel{r=x^{4 / n}}{=} \frac{4}{n} \cdot \frac{\int_{0}^{1} \frac{x^{(4 / n)+1} d x}{\sqrt{1-x^{4}}}}{\int_{0}^{1} \frac{x^{(4 / n)-1} d x}{\sqrt{1-x^{4}}}} \\
&=y\left(\frac{4}{n}-1\right)=\frac{16}{n^{2}} \frac{1}{y\left(\frac{4}{n}+1\right)} .
\end{aligned}
$$

This observation leads to formulas for Euler's continued fractions $[\mathbf{1 6}$, p. $36(25)]$.

Corollary 8. For every positive integer $m$

$$
\begin{aligned}
& y(4 m+1)=\left[\frac{2 \cdot 4 \cdot \ldots \cdot(2 m)}{1 \cdot 3 \cdot \ldots \cdot(2 m-1)}\right]^{2} \frac{4}{\pi}, \\
& y(4 m-1)=\left[\frac{1 \cdot 3 \cdot \ldots \cdot(2 m-1)}{2 \cdot 4 \cdot \ldots \cdot(2 m)}\right]^{2} 4 m^{2} \pi .
\end{aligned}
$$


Proof: Let $n=1 / m$ in (42). Then combining the above formula with (36) and (25) we obtain

$$
\begin{aligned}
y(4 m+1) & =4 m \cdot \frac{\int_{0}^{1} \frac{d r}{\sqrt{1-r^{n}}}}{\int_{0}^{1} \frac{r^{n / 2} d r}{\sqrt{1-r^{n}}}} \\
& =(4 m+2) \frac{\int_{0}^{1} \frac{x^{2 m+1} d x}{\sqrt{1-x^{2}}}}{\int_{0}^{1} \frac{x^{2 m} d x}{\sqrt{1-x^{2}}}}=\frac{4}{\pi}\left[\frac{2 \cdot 4 \cdot \ldots \cdot(2 m)}{1 \cdot 3 \cdot \ldots \cdot(2 m-1)}\right]^{2},
\end{aligned}
$$

which also gives $[\mathbf{1 6}$, p. $36(26)]$

$$
y(4 m-1)=\frac{(4 m)^{2}}{y(4 m+1)}=\left[\frac{1 \cdot 3 \cdot \ldots \cdot(2 m-1)}{2 \cdot 4 \cdot \ldots \cdot(2 m)}\right]^{2} 4 m^{2} \pi .
$$

Formula (41) implies

$$
\frac{\int_{0}^{1} \frac{d x}{\sqrt{1-x^{4}}}}{\int_{0}^{1} \frac{x^{2} d x}{\sqrt{1-x^{4}}}}=2+\underset{n=1}{\mathbf{K}}\left(\frac{(2 n-1)^{2}}{4}\right)=2.1884396152264 \ldots
$$

which is an analogue of Brouncker's formula for the lemniscate of Bernoulli. It also explains an "impossible relation"

$$
\underset{n=1}{\stackrel{\infty}{\mathbf{K}}}\left(\frac{(2 n-1)^{2}}{0}\right)=\frac{1}{2+{\underset{n=1}{\mathbf{K}}}_{n=1}^{\infty}\left(\frac{(2 n-1)^{2}}{4}\right)}
$$

established by Brouncker. One should only define

$$
{\underset{n}{\mathbf{K}}}_{n=1}^{\infty}\left(\frac{(2 n-1)^{2}}{0}\right)=\lim _{s \rightarrow 0}(1+s) \frac{\int_{0}^{1} \frac{x^{s+2} d x}{\sqrt{1-x^{4}}}}{\int_{0}^{1} \frac{x^{s} d x}{\sqrt{1-x^{4}}}}=\frac{\int_{0}^{1} \frac{x^{2} d x}{\sqrt{1-x^{4}}}}{\int_{0}^{1} \frac{d x}{\sqrt{1-x^{4}}}} .
$$

The moment of inertia of the lemniscate of Bernoulli (as well as its length) can be calculated similarly to the length of the unit circle with 
the help of

$$
\begin{aligned}
y(4 n) & =\frac{3^{2}}{1 \cdot 5} \cdot \frac{7^{2}}{5 \cdot 9} \cdot \ldots \cdot \frac{(4 n-1)^{2}}{(4 n-3)(4 n+1)} \cdot y(0)(4 n+1), \\
y(4 n+2) & =\frac{1 \cdot 5}{3^{2}} \cdot \frac{5 \cdot 9}{7^{2}} \cdot \ldots \cdot \frac{(4 n-3)(4 n+1)}{(4 n-1)^{2}} \cdot \frac{(4 n+1)}{y(0)} .
\end{aligned}
$$

It follows that the irrational numbers $y(1)=4 / \pi$ and $y(0)=1 / y(2)$ determine all values of $y(n)$ at positive integers $n$.

Finally, Euler's formula leads to a simple proof of Ramanujan's formula

$$
s+{\underset{n=1}{K}}_{n=1}^{\infty}\left(\frac{(2 n-1)^{2}}{2 s}\right)=4\left[\frac{\Gamma\left(\frac{3+s}{4}\right)}{\Gamma\left(\frac{1+s}{4}\right)}\right]^{2}, \quad s>0,
$$

see $[\mathbf{1 6}, \S 8$, p. 36], which we consider later. Here $\Gamma(x)$ is the Gamma function defined by

$$
\Gamma(x)=\lim _{k \rightarrow \infty} \frac{k ! k^{x-1}}{x(x+1) \ldots(x+k-1)},
$$

see $[\mathbf{2}, \S 1.1$, p. 3], [23, 12.11]. Motivated by Wallis' formula, Euler represented the infinite product on the left-hand side of Brouncker's formula in Theorem 5 as a quotient of two beta functions. This makes it quite possible that Brouncker's theorem led Euler to the discovery of beta and gamma functions. Notice a striking similarity in functional equations defining Brouncker's function $y(s)$ and the Gamma function:

$$
\begin{aligned}
y(s) y(s+2) & =(s+1)^{2} ; \\
s \Gamma(s) & =\Gamma(s+1) .
\end{aligned}
$$

\section{Brouncker's method for decimal places of $\pi$}

When Huygens learned Wallis' and Brouncker's formulas he couldn't believe them and asked for numerical confirmations. The rate of convergence of Wallis' product is very slow. The same is true for Brouncker's continued fraction, which is not a big surprise since it is the same as for telescopic series (7). To perform this calculation Brouncker derived from (13) important formulas (compare them with (43))

$$
\begin{aligned}
& y(4 n+1)=\frac{2^{2}}{1 \cdot 3} \cdot \frac{4^{2}}{3 \cdot 5} \cdot \ldots \cdot \frac{(2 n)^{2}}{(2 n-1)(2 n+1)} \cdot \frac{4}{\pi} \cdot(2 n+1), \\
& y(4 n+3)=\frac{1 \cdot 3}{2^{2}} \cdot \frac{3 \cdot 5}{4^{2}} \cdot \ldots \cdot \frac{(2 n-1)(2 n+1)}{(2 n)^{2}} \cdot(2 n+1) \pi .
\end{aligned}
$$


If $n=6$, then the continued fraction $y(4 \cdot 6+1)=y(25)$ has partial denominators equal $2 \cdot 25=50$, which considerably improves its convergence. Thus we obtain the following boundaries for $\pi$

$$
\beta_{0} \cdot \frac{Q_{2 k+1}}{P_{2 k+1}}<\pi<\beta_{0} \cdot \frac{Q_{2 k}}{P_{2 k}}
$$

where

$$
\beta_{0}=4 \cdot \frac{2^{2} \cdot 4^{2} \cdot 6^{2} \cdot 8^{2} \cdot 10^{2} \cdot 12^{2}}{3^{2} \cdot 5^{2} \cdot 7^{2} \cdot 9^{2} \cdot 11^{2}}=78.602424992035381646 \ldots
$$

and $P_{j} / Q_{j}$ are convergents to $y(25)$. Putting $k=0,1,2$ in the above formula we find that

$$
\begin{aligned}
& k=0, \quad 3.14158373269526<\pi<3.14409699968142 ; \\
& k=1, \quad 3.14159265194782<\pi<3.14159274082066 ; \\
& k=2, \quad 3.14159265358759<\pi<3.14159265363971 .
\end{aligned}
$$

Notice that already the first convergent to $y(25)$ gives four true places of $\pi$. The fifth convergent without tedious calculations gives eleven true places. This was the first algebraic calculation of $\pi$. Viète in 1593 couldn't use his formula and instead applied the traditional method of Archimedes to obtain 9 decimal places. In 1596 Ludolph van Ceulen obtained 20 decimal places by using a polygon with $60 \times 2^{29}$ sides. The amount of calculations made by Ludolph is incomparable with short and beautiful Brouncker's calculations. A detailed historical report on Brouncker's calculations can be found in [18]. It looks like that this Brouncker's achievement remained unnoticed and even his formulas (47) were later rediscovered by Euler.

\section{Brouncker's continued fractions for sinusoidal spirales}

Assuming that $n$ is a positive integer, let us consider the length of an $n$-lemniscate as an infinite product as well as a quotient of two integrals (37). These formulas resemble the formulas for the unit circle and give some hopes to extend Brouncker's formula to $n$-lemniscates. 
Applying (31) to a function

$$
\begin{aligned}
y(s) & =(q+s) \frac{\int_{0}^{1} \frac{x^{r+s+q-1} d x}{\sqrt{1-x^{2 r}}}}{\int_{0}^{1} \frac{x^{r+s-q-1} d x}{\sqrt{1-x^{2 r}}}} \\
& =(q+s) \prod_{j=0}^{\infty} \frac{(r+s-q+2 j r)(2 r+s+q+2 j r)}{(r+s+q+2 j r)(2 r+s-q+2 j r)},
\end{aligned}
$$

we obtain

$$
\begin{aligned}
y(s) & =(q+s) \cdot \frac{(r+s-q) \cdot(2 r+s+q)}{(r+s+q) \cdot(2 r+s-q))} \cdot \frac{(3 r+s-q) \cdot(4 r+s+q)}{(3 r+s+q) \cdot(4 r+s-q)} \cdot \ldots \\
& =(q+s) \cdot \frac{r+s-q}{r+s+q} \cdot \frac{1}{\frac{(r+(r+s)-q) \cdot(2 r+(s+r)+q)}{(r+(r+s)+q) \cdot(2 r+(r+s)-q)}} \cdot \ldots \\
& =(q+s)(s+r-q) \frac{1}{y(s+r)} .
\end{aligned}
$$

It follows that

$$
y(s) y(s+r)=(s+q)(s+r-q)
$$

which coincides with (13) for $q=1, r=2$. Moreover,

$$
s(s+r)=s^{2}+s r<s^{2}+s r+q(r-q)=(s+q)(s+r-q),
$$

if $r>q$. Hence the basic principles of Brouncker's method are valid.

Having got (49) we forget for a time being the formula for $y$ and assume that $y(s)$ is just a positive function on $(0,+\infty)$ satisfying $(49)$ and $s<y(s)$ for $s>0$. Then

$$
s<y(s)=\frac{(s+q)(s+r-q)}{y(s+r)}<\frac{(s+q)(s+r-q)}{s+r}=s+\frac{q(r-q)}{s+r} .
$$

Keeping in mind Brouncker's experience for the circle, we may directly develop $y(s)$ into a continued fraction skipping the intermediate step of an asymptotic expansion. Let

$$
y(s)=s+\frac{a_{0}}{y_{1}(s)} .
$$

To find $a_{1}$ we substitute this expression in (49) and obtain that

$$
\frac{a_{0}(s+r)}{y_{1}(s)}+\frac{a_{0} s}{y_{1}(s+r)}+\frac{a_{0}^{2}}{y_{1}(s) y_{1}(s+r)}=q(r-q) \text {. }
$$


If $y_{1}(s) \sim 2 s$ as $s \rightarrow+\infty$, then the equation shows that $a_{1}=q(r-q)$. We also obtain the functional equation for $y_{1}$

$$
y_{1}(s) y_{1}(s+r)=s y_{1}(s)+(s+r) y_{1}(s+r)+q(r-q) .
$$

Repeating the above arguments with

$$
y_{1}(s)=2 s+\frac{a_{1}}{y_{2}(s)},
$$

we find that

$$
\begin{aligned}
a_{1} & =2 r^{2}+q(r-q)=r(r+q)+r^{2}-q^{2}=(r+q)(2 r-q) \\
y_{2}(s) y_{2}(s+r) & =(s-r) y_{2}(s)+(s+2 r) y_{2}(s+r)+(r+q)(2 r-q) .
\end{aligned}
$$

Running the process by induction, we obtain a sequence of parameters and a sequence of functions satisfying

$$
\begin{aligned}
y_{k}(s) & =2 s+\frac{a_{k}}{y_{k+1}(s)}, \quad a_{k}=(k r+q)(k r+r-q) ; \\
y_{k}(s) y_{k}(s+r)= & (s-k r+r) y_{k}(s)+(s+k r) y_{k}(s+r) \\
& +(k r-r+q)(k r-q) .
\end{aligned}
$$

It follows that for every $k$ we have

(50) $y(s)=s+\frac{q(r-q)}{2 s}+\frac{(r+q)(2 r-q)}{2 s}{ }_{+\cdots+} \frac{(k r-r+q)(k r-q)}{y_{k}(s)}$.

Leaving to the next section the technical details, we pass to the limit in the above formula and obtain the following theorem by Euler.

Theorem 9 (Euler, [7, Section 47]). For positive $s$ and $q$ and for $r>q$

(51) $s+\underset{k=0}{\infty}\left(\frac{(k r+q)(k r+r-q)}{2 s}\right)=(q+s) \frac{\int_{0}^{1} x^{r+s+q-1}\left(1-x^{2 r}\right)^{-1 / 2} d x}{\int_{0}^{1} x^{r+s-q-1}\left(1-x^{2 r}\right)^{-1 / 2} d x}$.

Euler's Theorem for $s=q=1 / 2$ combined with (37) implies an extension of Brouncker's formula for the $2 r$-lemniscate

$$
\frac{2}{\int_{0}^{1} \frac{d x}{\sqrt{1-x^{2 r}}}}=1+\underset{k=1}{\infty}\left(\frac{(2(k-1) r+1)(2 k r-1)}{2}\right) .
$$

For $r=1$ we obtain Brouncker's formula, for $r=2$ the formula for the lemniscate of Bernoulli

$$
\frac{2}{\int_{0}^{1} \frac{d x}{\sqrt{1-x^{4}}}}=1+\frac{1 \cdot 3}{2}+\frac{5 \cdot 7}{2}+\frac{9 \cdot 11}{2}+\frac{13 \cdot 15}{2}+\cdots
$$


and for Kiepert's curve (Figure 3, $\mathrm{r}=3$ ) we have

$$
\frac{2}{\int_{0}^{1} \frac{d x}{\sqrt{1-x^{6}}}}=1+\frac{1 \cdot 5}{2}+\frac{7 \cdot 11}{2}+\frac{13 \cdot 17}{2}+\frac{19 \cdot 23}{2}+\cdots
$$

\section{A rigorous approach to Brouncker's proof}

Since Brouncker's continued fraction is a partial case of the BrounckerEuler continued fraction (51) for $r=2$ and $q=1$, we give the proof for the general case of Theorem 9 . We keep the notations introduced in the previous section

$$
a_{k}=(k r+q)(k r+r-q), \quad k=0,1,2, \ldots .
$$

To transform the incomplete induction in Brouncker's proof to complete we need the Euler-Wallis formulas $[\mathbf{1 0},(2.1 .6)]$ for convergents $P_{n} / Q_{n}$ of continued fractions (51), which in the case under consideration look as follows

$$
\begin{array}{lll}
P_{n}(s)=2 s P_{n-1}(s)+a_{n-1} P_{n-2}(s), & P_{0}(s)=s, & P_{-1}(s)=1, \\
Q_{n}(s)=2 s Q_{n-1}(s)+a_{n-1} Q_{n-2}(s), & Q_{0}(s)=1, & Q_{-1}(s)=0,
\end{array}
$$

and the determinant identity, which follows from them $[\mathbf{1 0},(2.1 .9)]$

$$
P_{n+1}(s) Q_{n}(s)-P_{n}(s) Q_{n+1}(s)=(-1)^{n} a_{0} a_{1} \ldots a_{n} .
$$

Lemma 10. The polynomials $P_{n}$ and $Q_{n}$ have the following algebraic structure

$$
P_{n}(s)=\left\{\begin{array}{ll}
p_{n}\left(s^{2}\right) & \text { if } n=2 k+1 ; \\
s p_{n}\left(s^{2}\right) & \text { if } n=2 k .
\end{array} \quad Q_{n}(s)= \begin{cases}s q_{n}\left(s^{2}\right) & \text { if } n=2 k+1 ; \\
p_{n}\left(s^{2}\right) & \text { if } n=2 k .\end{cases}\right.
$$

Proof: It follows from the Euler-Wallis formulas.

By Lemma 10 convergents to the Brouncker-Euler continued fractions are all odd:

$$
\frac{P_{n}(-s)}{Q_{n}(-s)}=-\frac{P_{n}(s)}{Q_{n}(s)}
$$

This, by the way, shows that (13) cannot hold for $s<0$. There are interesting concealed reasons for that. We discuss them later.

Lemma 11. The values of $P_{n}(s)$ at $s=q$ and $s=r-q$ are given by the products

$$
\begin{aligned}
P_{n}(q) & =q(q+r) \ldots(q+n r) ; \\
P_{n}(r-q) & =(r-q)(2 r-q) \ldots(n r+r-q) .
\end{aligned}
$$


Proof: Since $P_{0}(s)=s$, both formulas hold for $n=0$. Assuming that they hold for $P_{0}, P_{1}, \ldots, P_{n}$, we have by the Euler-Wallis formulas

$$
\begin{aligned}
P_{n+1}(q) & =2 q P_{n}(q)+(n r+q)(n r+r-q) P_{n-1}(q) \\
& =P_{n-1}(q)\{2 q(q+n r)+(n r+q)(n r+r-q)\} \\
& =P_{n-1}(q)(q+n r)(q+(n+1) r)
\end{aligned}
$$

and similarly

$$
\begin{aligned}
P_{n+1}(r-q) & =2(r-q) P_{n}(r-q)+(n r+q)(n r+r-q) P_{n-1}(r-q) \\
& =P_{n-1}(q)\{2(r-q)(n r+r-q)+(n r+q)(n r+r-q)\} \\
& =P_{n-1}(r-q)(n r+r-q)((n+1) r+r-q) .
\end{aligned}
$$

Theorem 12. For $n=0,1, \ldots$

$$
P_{n}(s) P_{n}(s+r)-(s+q)(s+r-q) Q_{n}(s) Q_{n}(s+r)=(-1)^{n+1} \prod_{j=0}^{n} a_{j} .
$$

Proof: Since $P_{0}(s)=s$ and $Q_{0}(s) \equiv 1$, we see that

$$
s(s+r)-(s+q)(s+r-q)=-q(r-q),
$$

which proves (57) for $n=0$. By (53) we have

$$
\begin{aligned}
& \frac{P_{n+1}(s)}{Q_{n+1}(s)} \cdot \frac{P_{n+1}(s+r)}{Q_{n+1}(s+r)}-(s+q)(s+r-q) \\
&=\frac{P_{n}(s)}{Q_{n}(s)} \cdot \frac{P_{n}(s+r)}{Q_{n}(s+r)}-(s+q)(s+r-q) \\
&+\left\{\frac{P_{n+1}(s)}{Q_{n+1}(s)}-\frac{P_{n}(s)}{Q_{n}(s)}\right\} \frac{P_{n+1}(s+r)}{Q_{n+1}(s+r)} \\
&+\left\{\frac{P_{n+1}(s+r)}{Q_{n+1}(s+r)}-\frac{P_{n}(s+r)}{Q_{n}(s+r)}\right\} \frac{P_{n}(s)}{Q_{n}(s)} \\
&=-\frac{(-1)^{n} a_{0} \ldots a_{n}}{Q_{n}(s) Q_{n}(s+r)}+\frac{(-1)^{n} a_{0} \ldots a_{n}}{Q_{n+1}(s) Q_{n}(s)} \frac{P_{n+1}(s+r)}{Q_{n+1}(s+r)} \\
&+\frac{(-1)^{n} a_{0} \ldots a_{n}}{Q_{n+1}(s+r) Q_{n}(s+r)} \frac{P_{n}(s)}{Q_{n}(s)} .
\end{aligned}
$$

The idea of these transforms is to prove by induction that the left hand side of (58) is $O\left(1 / s^{2 n+2}\right)$ as $s \rightarrow+\infty$ and find the coefficient at $1 / s^{2 n+2}$. 
By (53)

$$
\frac{P_{n+1}(s+r)}{Q_{n+1}(s+r)}=\frac{P_{1}(s+r)}{Q_{1}(s+r)}+O\left(\frac{1}{s^{3}}\right)=s+r+\frac{a_{0}}{2 s}+O\left(\frac{1}{s^{2}}\right) .
$$

Substituting this in (58) we obtain

$$
\begin{aligned}
\frac{(-1)^{n}}{a_{0} \ldots a_{n}}\left\{\frac{P_{n+1}(s)}{Q_{n+1}(s)}\right. & \left.\cdot \frac{P_{n+1}(s+r)}{Q_{n+1}(s+r)}-(s+q)(s+r-q)\right\} \\
= & \frac{-1}{Q_{n}(s) Q_{n}(s+r)}+\frac{s+r+a_{0} /(2 s)}{Q_{n+1}(s) Q_{n}(s)} \\
& +\frac{s+a_{0} /(2 s)}{Q_{n+1}(s+r) Q_{n}(s+r)}+O\left(\frac{1}{s^{2 n+3}}\right) .
\end{aligned}
$$

To continue the calculations we need a technical lemma.

Lemma 13. The polynomials $Q_{n}(s)$ satisfy

$$
\frac{Q_{n}(s+r)}{(2 s)^{n}}=1+\frac{n r}{s}+O\left(\frac{1}{s^{2}}\right), \quad s \rightarrow+\infty .
$$

Proof: By the Euler-Wallis formulas

$$
\begin{aligned}
\frac{Q_{n}(s+r)}{(2 s)^{n}} & =\left(1+\frac{r}{s}\right) \frac{Q_{n-1}(s+r)}{(2 s)^{n-1}}+\frac{a_{n-1} Q_{n-2}(s+r)}{(2 s)^{n}} \\
& =\left(1+\frac{r}{s}\right) \frac{Q_{n-1}(s+r)}{(2 s)^{n-1}}+O\left(\frac{1}{s^{2}}\right)=\cdots \\
& =\left(1+\frac{r}{s}\right)^{n}+O\left(\frac{1}{s^{2}}\right)=1+\frac{n r}{s}+O\left(\frac{1}{s^{2}}\right),
\end{aligned}
$$

as stated.

Putting $r=0$ in (60), we obtain

$$
\frac{Q_{n}(s)}{(2 s)^{n}}=1+O\left(\frac{1}{s^{2}}\right) .
$$

Applying (60) we find that

$$
\begin{aligned}
-\frac{1}{Q_{n}(s) Q_{n}(s+r)} & =-\frac{1}{(2 s)^{2 n}} \frac{1}{1+\frac{n r}{s}+O\left(\frac{1}{s^{2}}\right)} \cdot \frac{1}{1+O\left(\frac{1}{s^{2}}\right)} \\
& =-\frac{1}{(2 s)^{2 n}} \cdot\left(1-\frac{n r}{s}\right)+O\left(\frac{1}{s^{2 n+2}}\right) .
\end{aligned}
$$


For the second term in the right hand side of (59) we have

$$
\begin{aligned}
\frac{s+r+a_{0} /(2 s)}{Q_{n+1}(s) Q_{n}(s)} & =\frac{1}{2} \cdot \frac{1+r / s+a_{0} /\left(2 s^{2}\right)}{(2 s)^{2 n}} \cdot \frac{1}{1+O\left(\frac{1}{s^{2}}\right)} \cdot \frac{1}{1+O\left(\frac{1}{s^{2}}\right)} \\
& =\frac{1}{2} \cdot \frac{1}{(2 s)^{2 n}} \cdot\left(1+\frac{r}{s}\right)+O\left(\frac{1}{s^{2 n+2}}\right) .
\end{aligned}
$$

Finally for the third term in (59) we obtain

$$
\begin{aligned}
& \frac{s+a_{0} /(2 s)}{Q_{n+1}(s+r) Q_{n}(s+r)} \\
& \quad=\frac{1}{2} \cdot \frac{1+a_{0} /\left(2 s^{2}\right)}{(2 s)^{2 n}} \cdot \frac{1}{1+\frac{(n+1) r}{s}+O\left(\frac{1}{s^{2}}\right)} \cdot \frac{1}{1+\frac{n r}{s}+O\left(\frac{1}{s^{2}}\right)} \\
& \quad=\frac{1}{2} \cdot \frac{1}{(2 s)^{2 n}} \cdot\left(1-\frac{(2 n+1) r}{s}\right)+O\left(\frac{1}{s^{2 n+2}}\right) .
\end{aligned}
$$

Combining (62)-(64) with (58), we obtain

$$
\frac{P_{n+1}(s)}{Q_{n+1}(s)} \cdot \frac{P_{n+1}(s+r)}{Q_{n+1}(s+r)}-(s+q)(s+r-q)=O\left(\frac{1}{s^{2 n+2}}\right)
$$

or equivalently

(65) $P_{n+1}(s) P_{n+1}(s+r)-(s+q)(s+r-q) Q_{n+1}(s) Q_{n+1}(s+r)=O(1)$,

as $s \rightarrow+\infty$. Since the left hand side of $(65)$ is a polynomial of degree $2 n+4$, the right hand side of (65) must be a constant. Its value can be obtained by putting $s=-q$ in (65) and by applying Lemmas 10 and 11

$$
\begin{aligned}
P_{n+1}(-q) P_{n+1}(r-q) & =(-1)^{n+2} P_{n+1}(q) P_{n+1}(r-q) \\
& =(-1)^{n+2} a_{0} \ldots a_{n+1} .
\end{aligned}
$$

Corollary 14. For $k=0,1,2, \ldots$ and $s>0$

(66) $\frac{P_{2 k}(s)}{Q_{2 k}(s)} \frac{P_{2 k}(s+r)}{Q_{2 k}(s+r)}<(s+q)(s+r-q)<\frac{P_{2 k+1}(s)}{Q_{2 k+1}(s)} \frac{P_{2 k+1}(s+r)}{Q_{2 k+1}(s+r)}$.

Proof: The proof follows from (57) since for $s>0$ the left hand side of (57) is negative for even $n$ 's and is positive for for odd $n$ 's.

Now by (53) even convergents of any continued fractions with positive terms increase and odd convergents decrease. So, if one proves that any 
Brouncker-Euler continued fraction converges, then by Corollary 14 the limit $y(s)$ of the convergents must satisfy

$$
y(s) y(s+r)=(s+r)(s+r-q), \quad s<y(s),
$$

which completes the proof of Theorem 9 .

Possibly the easiest way to prove the convergence is to apply a simple old theorem by Pringsheim [17], see [11, Ch. I, §5].

Theorem 15. Let $p_{n}>0, q_{n}>0$ and

$$
\sum_{n=1}^{\infty}\left(\frac{q_{n-1} q_{n}}{p_{n}}\right)^{1 / 2}=+\infty .
$$

Then $q_{0}+\underset{n=1}{\mathbf{K}}\left(\frac{p_{n}}{q_{n}}\right)$ converges.

In our case $q_{n}=2 s$ and $p_{n}=((n-1) r+q)(n r-q)$. Therefore the continued fraction (51) converges. Theorem 15 is a kind of theorem, which summarizes explicitly the arguments used indirectly by previous authors. Also, one may notice that the equivalence transforms reduce (21) to the form with $q_{n}=2, p_{n}=(2 n-1)^{2} / s^{2}$, which from the point of view of convergence does not differ much from the Brouncker-Euler continued fractions. Recall that the convergence of Brouncker's continued fraction was established in Section 2.

\section{The limit case of Theorem 9}

The change of variables $r:=r q, s:=s q$ followed by the change of variables $x=y^{1 / q r}$ transforms (51) into

$$
\begin{aligned}
\frac{1}{2 s}+\frac{(r+1)(2 r-1)}{2 s} & +\frac{(2 r+1)(3 r-1)}{2 s}+\frac{(3 r+1)(4 r-1)}{2 s}+\cdots \\
= & \frac{1}{r-1} \cdot \frac{(1+s) \int_{0}^{1} \frac{y^{\frac{s+1}{r}} d y}{\sqrt{1-y^{2}}}-s \int_{0}^{1} \frac{y^{\frac{s-1}{r}} d y}{\sqrt{1-y^{2}}}}{\int_{0}^{1} \frac{y^{\frac{s-1}{r}} d y}{\sqrt{1-y^{2}}}}
\end{aligned}
$$

Observing that the even convergents of any positive continued fraction are smaller and the odd convergent are greater than the continued fraction, and applying l'Hôpital's rule to find the limit as $r \rightarrow 1+$, we obtain 
a formula

$$
\begin{gathered}
\frac{1}{2 s}+\frac{1 \cdot 2}{2 s}+\frac{2 \cdot 3}{2 s}+\frac{3 \cdot 4}{2 s}+\cdots \\
=\frac{(s+1)^{2} \int_{0}^{1} \frac{y^{s+1} \ln \frac{1}{y} d y}{\sqrt{1-y^{2}}}-s(s-1) \int_{0}^{1} \frac{y^{s-1} \ln \frac{1}{y} d y}{\sqrt{1-y^{2}}}}{\int_{0}^{1} \frac{y^{s-1} d y}{\sqrt{1-y^{2}}}}
\end{gathered}
$$

interpolating formula (11) at $s=1$ :

$$
\frac{1}{2}+\frac{1 \cdot 2}{2}+\frac{2 \cdot 3}{2}+\frac{3 \cdot 4}{2}+\cdots=\frac{4 \int_{0}^{1} \frac{y^{2} \ln \frac{1}{y} d y}{\sqrt{1-y^{2}}}}{\int_{0}^{1} \frac{d y}{\sqrt{1-y^{2}}}}=\log 4-1 .
$$

By parts integration and Lemma 3 show that

(67) $\int_{0}^{1} \frac{t^{s+1} \ln \frac{1}{t} d t}{\sqrt{1-t^{2}}}=\frac{s}{s+1} \int_{0}^{1} \frac{t^{s-1} \ln \frac{1}{t} d t}{\sqrt{1-t^{2}}}-\frac{1}{(s+1)^{2}} \int_{0}^{1} \frac{t^{s-1} d t}{\sqrt{1-t^{2}}}$,

which results in an interesting formula

$$
y(s)=\frac{1}{2 s}+\frac{1 \cdot 2}{2 s}+\frac{2 \cdot 3}{2 s}+\frac{3 \cdot 4}{2 s}+\cdots=2 s \frac{\int_{0}^{1} \frac{t^{s-1} \ln \frac{1}{t} d t}{\sqrt{1-t^{2}}}}{\int_{0}^{1} \frac{t^{s-1} d t}{\sqrt{1-t^{2}}}}-1 .
$$

Applying (67) and Lemma 3 again, we obtain by (68) that $y(s)$ satisfies the functional equation

$$
\frac{y(s)}{s}-\frac{y(s+2)}{s+2}=\frac{2}{s(s+1)(s+2)} .
$$

Iterating (69), we obtain

(70) $\frac{1}{2 s}+\frac{1 \cdot 2}{2 s}+\frac{2 \cdot 3}{2 s}+\cdots=\sum_{k=0}^{\infty} \frac{2 s}{(s+2 k)(s+2 k+1)(s+2 k+2)}$,

which shows that $\lim _{s \rightarrow 0+} y(s)=1$. Elementary algebra and (70) result in

$$
\pi=2+\frac{16}{1 \cdot 3 \cdot 5}+\frac{16}{5 \cdot 7 \cdot 9}+\frac{16}{9 \cdot 11 \cdot 13}+\frac{16}{13 \cdot 15 \cdot 17}+\cdots .
$$




\section{Complex analysis approach to Brouncker's proof}

An explanation of violation of (13) for negative $s$ can be easily found if we leave the real line and make $s$ be complex $z=s+i t$. The key result here is Van Vleck's theorem proved in 1901 [20], [10, Theorem 4.29].

Theorem 16. Let the partial denominators $b_{n}$ of a continued fraction $\stackrel{\infty}{K}_{n=1}^{\infty}\left(\frac{1}{b_{n}}\right)$ satisfy

$$
-\frac{\pi}{2}+\varepsilon<\arg b_{n}<\frac{\pi}{2}-\varepsilon \quad n=1,2, \ldots,
$$

where $\varepsilon$ can be arbitrary small positive number. Then

(a) the $n$-th convergent $f_{n}$ of the continued fraction is finite and satisfies

$$
-\frac{\pi}{2}+\varepsilon<\arg f_{n}<\frac{\pi}{2}-\varepsilon \quad n=1,2, \ldots ;
$$

(b) both limits $\lim _{k} f_{2 k}$ and $\lim _{2 k+1}$ exist and are finite;

(c) the continued fraction converges if $\sum_{n}\left|b_{n}\right|=+\infty$;

(d) if the continued fraction converges to $f$, then $f$ is finite and $|\arg f| \leqslant \pi / 2$.

Any continued fraction $\underset{n=1}{\mathbf{K}}\left(\frac{p_{n}}{q_{n}}\right)$ with positive terms is equivalent (= has the same convergents) to the type of continued fraction considered in Van Vleck's Theorem:

$$
\frac{1}{\frac{1}{p_{1}} q_{1}}+\frac{1}{\frac{p_{1}}{p_{2}} q_{2}}+\frac{1}{\frac{p_{2}}{p_{1} p_{3}} q_{3}}+\frac{1}{\frac{p_{1} p_{3}}{p_{2} p_{4}} q_{4}}+\frac{1}{\frac{p_{2} p_{4}}{p_{1} p_{3} p_{5}} q_{5}}+\cdots .
$$

For Brouncker's continued fraction $q_{n}=2 z, p_{n}=(2 n-1)^{2}$ and for more general Euler's continued fraction (51) $p_{n+1}=(k n+q)(k n+r-q)$. The convergence of the Brouncker-Euler continued fraction for $\Re(z)=s>0$ follows by Theorem 16

$$
\sum_{n} u_{n}+\sum_{n} v_{n}=\sum_{n} \frac{p_{1} p_{3} \ldots p_{2 n-1}}{p_{2} p_{4} \ldots p_{2 n}}+\sum_{n} \frac{p_{2} p_{4} \ldots p_{2 n}}{p_{1} p_{3} \ldots p_{2 n+1}}=+\infty,
$$

since $2 /(2 n+1)=2 \sqrt{u_{n} \cdot v_{n}} \leqslant u_{n}+v_{n}$. By Theorem $16 \Re P_{n}(z) / Q_{n}(z)>0$ for $\Re z>0$. By (54) this implies that all zeros of $P_{n}$ and $Q_{n}$ are located on the imaginary axis. These zeros make a barrier for an analytic continuation of Brouncker's continued fraction to the left half-plane but they do not influence the corresponding infinite product (see Theorem 6) coinciding with the continued fraction in the right half plane. Hence Brouncker's continued fraction in the right half-plane extends analytically to a meromorphic function in $\mathbb{C}$ with poles at $-4 n+1, n=1,2, \ldots$. 


\section{Ramanujan formula and Brouncker orthogonal polynomials}

Ramanujan formula (46) for Brouncker's continued fraction is an elementary corollary of Brouncker's Theorem 6 and Euler's extension of Wallis' formula:

$$
\begin{aligned}
& s+{\underset{n=1}{\mathbf{K}}}_{1}\left(\frac{(2 n-1)^{2}}{2 s}\right)=(s+1) \frac{\int_{0}^{1} \frac{x^{s+2} d x}{\sqrt{1-x^{4}}}}{\int_{0}^{1} \frac{x^{s} d x}{\sqrt{1-x^{4}}}} \\
& =(s+1) \frac{\int_{0}^{1} x^{s+2}\left(1-x^{4}\right)^{-1 / 2} d x}{\int_{0}^{1} x^{s}\left(1-x^{4}\right)^{-1 / 2} d x} \\
& \stackrel{x=t^{1 / 4}}{=}(s+1) \frac{\int_{0}^{1} t^{(s+3) / 4-1}(1-t)^{1 / 2-1} d t}{\int_{0}^{1} t^{(s+1) / 4-1}(1-t)^{1 / 2-1} d t} \\
& =(s+1) \frac{B\left(\frac{s+3}{4}, \frac{1}{2}\right)}{B\left(\frac{s+1}{4}, \frac{1}{2}\right)} \\
& =(s+1) \frac{\Gamma\left(\frac{s+3}{4}\right) \Gamma\left(\frac{1}{2}\right) \Gamma\left(\frac{s+3}{4}\right)}{\Gamma\left(\frac{s+5}{4}\right) \Gamma\left(\frac{s+1}{4}\right) \Gamma\left(\frac{1}{2}\right)} \\
& =(s+1) \frac{\left[\Gamma\left(\frac{s+3}{4}\right)\right]^{2}}{\left(\frac{s+1}{4}\right) \Gamma\left(\frac{s+1}{4}\right) \Gamma\left(\frac{s+1}{4}\right)} \\
& =4\left[\frac{\Gamma\left(\frac{3+s}{4}\right)}{\Gamma\left(\frac{1+s}{4}\right)}\right]^{2} \text {. }
\end{aligned}
$$


Applying a well-known formula for the Gamma function $[\mathbf{2 3}, \S 12.14]$

$$
\Gamma(z) \Gamma(1-z)=\frac{\pi}{\sin \pi z}
$$

with $z=1 / 4-i t / 4$, we obtain that

$$
\Gamma\left(\frac{3+i t}{4}\right)=\Gamma(1-z)=\frac{\pi}{\sin \pi z} \frac{1}{\Gamma(z)}
$$

and therefore

$$
\frac{1}{4}\left[\frac{\Gamma\left(\frac{1+i t}{4}\right)}{\Gamma\left(\frac{3+i t}{4}\right)}\right]^{2}=\frac{1-\cos 2 \pi z}{8 \pi^{2}}|\Gamma(z)|^{4}=\frac{1}{8 \pi^{2}}|\Gamma(z)|^{4}\left(1-i \sinh \frac{\pi t}{2}\right) .
$$

It follows that the inverse of Brouncker's continued fraction has positive real part in the right-half plane as it should be by Van Vleck's Theorem. Since $1 / y(s)=1 / s+o\left(1 / s^{2}\right)$ as $s \rightarrow+\infty$, the Stieltjes inversion formula implies that

$$
\frac{1}{8 \pi^{3}} \int_{-\infty}^{+\infty}\left|\Gamma\left(\frac{1+i t}{4}\right)\right|^{4} d t=1 .
$$

Moreover, since $P_{n}$ are the denominators of convergents to the reciprocal Brouncker continued fraction, by Chebychev's Theorem $[\mathbf{1 2}, \S 12]$ Brouncker's polynomials $P_{n}(i t)$ are orthogonal polynomials:

$$
\frac{1}{8 \pi^{3}} \int_{-\infty}^{+\infty} P_{n}(i t) t^{k}\left|\Gamma\left(\frac{1+i t}{4}\right)\right|^{4} d t=0, \quad 0 \leqslant k<n .
$$

For the maximal value of the weight of orthogonality we have by Theorem 6

$$
\begin{aligned}
\frac{1}{8 \pi^{2}}\left|\Gamma\left(\frac{1}{4}\right)\right|^{4} & =\frac{1}{y(0)}=\frac{1}{\lim _{s \rightarrow 0-} y(s)}=2+{\underset{n=1}{\mathbf{K}}}_{n=1}\left(\frac{(2 n-1)^{2}}{4}\right) \\
& =3 \prod_{n=1}^{\infty} \frac{(4 n-1)(4 n+3)}{(4 n+1)^{2}}=\prod_{n=1}^{\infty} \frac{(4 n-1)^{2}}{(4 n-3)(4 n+1)}
\end{aligned}
$$

which clarifies the meaning of the "impossible relation" (45).

Notice that the convergence of Brouncker's continued fraction in the right-half plane (even the convergence for $s=1$ is enough) means that the moment problem associated with the Brouncker weight is determined. 
Formula (73) is a partial case of Wilson's formula

$$
\begin{array}{r}
\frac{1}{2 \pi} \int_{0}^{\infty}\left|\frac{\Gamma(a+i x) \Gamma(b+i x) \Gamma(c+i x) \Gamma(d+i x)}{\Gamma(2 i x)}\right|^{2} d x \\
=\frac{\Gamma(a+b) \Gamma(a+c) \Gamma(a+d) \Gamma(b+c) \Gamma(b+d) \Gamma(c+d)}{\Gamma(a+b+c+d)}
\end{array}
$$

see $[\mathbf{2}$, p. 152]. To see this one should put $a=0, b=1 / 2, c=d=1 / 4$ and apply the duplication formula

$$
2^{2 z-1} \Gamma(z) \Gamma\left(z+\frac{1}{2}\right)=\sqrt{\pi} \Gamma(2 z)
$$

see [23, p. 240]. This by the way implies that Brouncker's polynomials are Wilson's polynomials.

Acknowledgements. I am grateful to Mourad Ismail (University of Central Florida) for answering my question on possible relationship of Brouncker's polynomials with known special functions and to Christian Berg (Institute for Mathematical Sciences, Copenhagen) for a useful discussion. I am indebted to Jacqueline Stedall (the Queen's College in Oxford) for an important reference [18] showing that Brouncker knew Euler's continued fractions (43).

\section{References}

[1] M. G. Agnesi, "Instituzioni analitiche ad uso della gioventù italiana", vol. 1, Milan, 1748.

[2] G. E. Andrews, R. Askey And R. Roy, "Special functions", Encyclopedia of Mathematics and its Applications 71, Cambridge University Press, Cambridge, 1999.

[3] W. Brouncker, The squaring of the Hyperbola by an infinite series of rational numbers, Philos. Trans. Royal Soc. London 3 (1668), 645-649.

[4] J. J. O'Connor And E. F. Robertson, William Brouncker, MacTutor History of Mathematics, http://www-gap.dcs.st-and.ac.uk/ history/Mathematicians /Brouncker.html.

[5] H. M. EDWARDs, "Fermat's last theorem. A genetic introduction to algebraic number theory", Graduate Texts in Mathematics 50, Springer-Verlag, New York-Berlin, 1977.

[6] L. Euler, De Fractionibus Continuus, Dissertatio, Commentarii Academiae Scientiarum Imperials Petropolitane, vol. IX for 1737 (1744), 98-137. 
[7] L. Euler, De Fractionibus Continuus, Observationes, Commentarii Academiae Scientiarum Imperials Petropolitane, vol. XI for 1739 (1750), 32-81.

[8] L. EULER, "Introductio in Analysin Infinitorum", Lausanne, 1748.

[9] L. Euler, "Integral Calculus I", Moscow, GITTL, 1956; translated from Latin Edition, St. Petersbourg, 1768.

[10] W. B. Jones And W. J. Thron, "Continued Fractions. Analytic theory and applications", Encyclopedia of Mathematics and its Applications 11, Addison-Wesley Publishing Co., Reading, Mass., 1980 .

[11] A. N. KhovanskiI, "Applications of continued fractions anf their generalizations to some problems of Numerical Analysis", GIITL, Moscow, 1956.

[12] S. Khrushchev, Continued fractions and orthogonal polynomials on the unit circle, J. Comput. Appl. Math. 178(1-2) (2005), $267-303$.

[13] A. N. Kolmogorov And A. P. Yushkevich, Eds., "History of Mathematics (Mathematics of XVII-th)" v. 2, Nauka, Moscow, 1970.

[14] F. D. Kramar, Integration methods of J. Wallis, (Russian), Istor.Mat. Issled. 14 (1961), 11-100.

[15] H. McKean And V. Moll, "Elliptic curves. Function theory, geometry, arithmetic", Cambridge University Press, Cambridge, 1997.

[16] O. Perron, "Die Lehre von den Kettenbrüchen. Dritte, verbesserte und erweiterte Aufl. Bd. II. Analytisch-funktionentheoretische Kettenbrüche”, B. G. Teubner Verlagsgesellschaft, Stuttgart, 1957.

[17] A. Pringsheim, Ueber ein Convergenz-Kriterium für die Kettenbrüche mit positiven Gleidern, Sitzungsber. der math.-phys Klasse der Kgl. Bayer. Akad. Wiss., München 29 (1899), 261-268.

[18] J. A. Stedall, Catching Proteus: the collaborations of Wallis and Brouncker I. Squaring the circle, Notes and Records Roy. Soc. London 54(3) (2000), 293-316.

[19] T. J. Stieltjes, Recherches sur les fractions continues, Ann. Fac. Sci. Toulouse Math. 8 (1894), J76-J122; 9 (1895), A5-A47; Russian translation; ONTI, Kiev.

[20] E. B. VAN VLECK, On the convergence of continued fractions with complex elements, Trans. Amer. Math. Soc. 2(3) (1901), 215-233.

[21] F. VIÈTE, "Variorum de rebus mathematicis responsorum liber VIII", Turonis, 1593.

[22] J. WALLIS, "The arithmetic of infinitesimals", Translated from the Latin and with an introduction by Jaequeline A. Stedall, Sources 
and Studies in the History of Mathematics and Physical Sciences, Springer-Verlag, New York, 2004.

[23] E. T. WhitTAKer AND G. N. WATSOn, "A course of modern analysis. An introduction to the general theory of infinite processes and of analytic functions; with an account of the principal transcendental functions", Reprint of the fourth (1927) edition, Cambridge Mathematical Library, Cambridge University Press, Cambridge, 1996.

Department of Mathematics

Atilim University

06836 Incek, Ankara

Turkey

E-mail address: svk_49@yahoo.com

Primera versió rebuda el 8 de febrer de 2005, darrera versió rebuda el 26 de setembre de 2005 . 